\title{
No marginal arbitrage of the second kind for high production regimes in discrete time production-investment models with proportional transaction costs
}

\author{
Bruno Bouchard * \\ Université Dauphine, CEREMADE, \\ and CREST-ENSAE \\ Paris, France \\ bouchard@ceremade.dauphine.fr
}

\author{
Adrien Nguyen Huu ${ }^{\dagger}$ \\ Université Dauphine, CEREMADE \\ and FiME- EDF R\&D \\ Paris, France \\ nguyen@ceremade.dauphine.fr
}

January 20, 2011

\begin{abstract}
We consider a class of production-investment models in discrete time with proportional transaction costs. For linear production functions, we study a natural extension of the no-arbitrage of the second kind condition introduced by M. Rásonyi [16]. We show that this condition implies the closedness of the set of attainable claims and is equivalent to the existence of a strictly consistent price system under which the evaluation of future production profits is strictly negative. This allows us to discuss the closedness of the set of terminal wealth in models with non-linear production, functions which may admit arbitrages of the second kind for low production regimes but not marginally for high production regimes.
\end{abstract}

Key words : financial markets with transaction costs, non-linear returns, no-arbitrage of the second kind, consistent price systems.

${ }^{*}$ This research is part of the Chair Finance and Sustainable Development sponsored by EDF and Calyon, and the Chair Les particuliers face au risque sponsored by Groupama.

${ }^{\dagger}$ Adrien Nguyen Huu expresses his gratitude to EDF R\&D and FiME Laboratoire de Finance des Marchés d'Énergies for its financial support. 


\section{Introduction}

This paper is motivated by applications in optimal hedging of electricity derivatives for electricity producers. Electricity producers sell derivative contracts that allow them to buy electricity at different periods and at a price fixed in advance. In practice, the producer can deliver the required quantities of electricity either by producing it or by buying it on the spot market. He can also try to cover himself through future contracts, but the granularity of the available maturities on the market is in general insufficient.

It is a typical situation where a financial agent can manage a portfolio by either trading on a financial market or by producing a good himself. Such models have already been studied in the literature, in particular by Bouchard and Pham [1] who discussed the questions of no-arbitrage, super-hedging and expected utility maximization in a discrete time model with proportional transaction costs, see also Kabanov and Kijima [7] and the references therein.

In [1], the assets are divided in two classes. The first class corresponds to purely financial assets, e.g. bonds, stocks, options, etc. The second class corresponds to industrial assets, e.g. plants or buildings. Industrial assets cannot be short-sold, contrary to financial assets. Moreover, they produce a (random) return at each period. These are expressed in terms of financial assets, and depend on the current inventory in industrial assets.

This model is well-adapted to industrial investment problems but not to production issues, since the production regime does not appear as a control.

In this paper, we consider another approach. As in [1], we work in a discrete time model with proportional transaction costs. Although it does not need to be explicit in the model, we have in mind that the assets are divided in two classes: the financial assets and industrial ones. Both can be traded in the market but some of them can be consumed in order to produce other assets. For instance, coal can be traded on the market, but can also be used to produce electricity in order to produce cash, once sold. The quantity used for production on the time period $[t, t+1]$ is chosen at time $t$. It leaves the portfolio and enters a production process. Depending on the quantity used, a (random) return enters the portfolio at time $t+1$. Therefore, the main difference with [1] is that we explicitly decide on the production level at each time step, rather than letting it implicitely be determined by inventories. 
Obviously, both approaches can be combined. We refrain from doing this in this paper in order to isolate the effect of our production model and to avoid unnecessary complexity.

As in [1], we first discuss the absence of arbitrage opportunity and its dual characterization. In [1], the authors adapt the notion of robust no-arbitrage introduced by Schachermayer [17]. It essentially means that there is still no-arbitrage even if transaction costs are slightly reduced and production returns are slightly increased. It should be noted that the arguments used in [1] could be easily adapted to our context. However, we prefer to adopt the (more natural) notion of no-arbitrage of the second kind, which was recently introduced in the context of financial markets with transactions costs by Rásonyi [16] under the name of no-sure gain in liquidation value, see also [4] for a continuous time version. The latter notion implies that we cannot turn an insolvent position at time $t$ into a position which is a.s. solvent at a later time $T$, by trading on the market. In models without transaction costs, this corresponds to the usual notion of no-arbitrage.

Another difference with Bouchard and Pham [1] is that we allow for reasonable arbitrages due to the production possibilities. Here, reasonable means that it may be possible to have a.s. positive net returns for low production regimes. However, they should be limited in the sense that marginal arbitrages for high production regimes are not possible.

The way we model this consists in assuming that the production function $\beta \rightarrow R(\beta)$ admits an affine upper bound $\beta \rightarrow c+L \beta$, which is somehow sharp for large values of $\beta$, and that the linear model in which $R$ is replaced by $L$ admits no arbitrage of the second kind. In the case where each component of $R$ is concave, the following may hold for $L$ and $R$ : $\lim _{\alpha \rightarrow \infty} R(\alpha \beta) / \alpha=L \beta$ (whenever it makes sense), i.e., noarbitrage holds in a marginal way for large regimes $\beta$. From the economic point of view, this means that gains can be made from production in reasonable situations, but that producing more becomes (marginally) risky when the regime of production is pushed too high.

From a mathematical point of view, it allows us to reduce the model to a linear one, at first, for which a nice dual formulation of the no-arbitrage condition is available. Indeed the set of dual variables can be fully described in terms of martingales evolving in appropriate sets. This is not the case for non-linear models (see [1]). The dual formulation is obtain by following the arguments of Rásonyi [16] which do not require 
to prove that the set of attainable claims is closed. One can then show that the set of attainable claims is indeed closed in probability both in the linear and the original models. As usual, this leads to a dual formulation of these sets. It can also be used to prove existence for expected utility maximization problems, which, in particular, allow the study of indifference prices.

Importantly, it should be noted that our approach is different from the notions of no marginal and no scalable arbitrage studied in [13] in the context of market models with convex trading cost functions, see also [14] and the references therein. We will discuss this in more detail in Remark 2.4 below.

We refer to [11] for a wide overview of models with proportional transaction costs. See also [14] and [15] for some more recent results in discrete time, and [5] or [6] for the continuous time setting.

The paper is organized as follows. We first describe our model, state the dual characterization of our no-arbitrage condition and important closedness properties in Section 2. Section 3 discusses applications to super-hedging and utility maximization problems. The proofs are collected in Section 4.

Notations: Unless otherwise specified, any element $x \in \mathbb{R}^{d}$ will be viewed as a column vector with entries $x^{i}, i \leq d$, and transposition is denoted by $x^{\prime}$ so that $x^{\prime} y$ stands for the scalar product. We write $\mathbb{M}^{d}$ to denote the set of square matrices $M$ of dimension $d$ with entries $M^{i j}, i, j \leq d$. The identity matrix is denoted by $I_{d}$. As usual, $\mathbb{R}_{+}^{d}$ and $\mathbb{R}_{-}^{d}$ stand for $[0, \infty)^{d}$ and $(-\infty, 0]^{d}$. The closure of a set $\Theta \subset \mathbb{R}^{n}$ is denoted by $\bar{\Theta}$, $n \geq 1$. We write cone $(\Theta)$ (resp. $\operatorname{conv}(\Theta))$ to denote the cone (resp. convex cone) generated by $\Theta$. Given a filtration $\mathbb{F}$ on a probability space $(\Omega, \mathcal{F}, \mathbb{P})$ and a set-valued $\mathcal{F}$-measurable family $A=\left(A_{t}\right)_{t \leq T}$, we denote by $L^{0}(A, \mathbb{F})$ the set of adapted processes $X=\left(X_{t}\right)_{t \leq T}$ such that $X_{t} \in A_{t} \mathbb{P}$ - a.s. for all $t \leq T$. For a $\sigma$-algebra $\mathcal{G}$ and a $\mathcal{G}$ measurable random set $A$, we write $L^{0}(A, \mathcal{G})$ for the collection of $\mathcal{G}$-measurable random variables that take values in $A \mathbb{P}$ - a.s. We similarly define the notations $L^{p}(A, \mathcal{G})$ for $p \in \mathbb{N} \cup \infty$, and simply write $L^{p}$ if $A$ and $\mathcal{G}$ are clearly given by the context. Unless otherwise specified, inequalities between random variables or inclusion between random sets have to be understood in the a.s. sense. 


\section{Definitions and main results}

\subsection{Model description}

From now on we denote by $T \in \mathbb{N} \backslash\{0\}$ a fixed time horizon and set $\mathbb{T}:=\{0,1, \ldots, T\}$. The complete filtration of the investor, $\mathbb{F}=\left(\mathcal{F}_{t}\right)_{t \in \mathbb{T}}$, is supported by a probability space $(\Omega, \mathcal{F}, \mathbb{P})$. We assume that $\mathcal{F}_{T}=\mathcal{F}$ and that $\mathcal{F}_{0}$ is trivial.

As in [17], we model exchange prices by an adapted process $\pi=\left(\pi_{t}\right)_{t \in \mathbb{T}}$ taking values in the set $\mathbb{M}^{d}$ of square $d$-dimensional matrices, for some $d \geq 1$, satisfying the following conditions for all $t \leq T$ and $i, j, k \leq d$ :

$$
\text { (i) } \pi_{t}^{i j}>0 \text {, (ii) } \pi_{t}^{i i}=1 \text {, (iii) } \pi_{t}^{i j} \pi_{t}^{j k} \geq \pi_{t}^{i k} .
$$

Here, $\pi_{t}^{i j}$ should be interpreted as the number of units of asset $i$ required to obtain one unit of asset $j$ at time $t$. The conditions (i) and (ii) need no comment. The third condition is also natural. It means that it is always cheaper to buy directly units of asset $k$ from units of asset $i$ rather then going through the asset $j$. Note that, combined with (ii), it implies that $\pi_{t}^{i j} \pi_{t}^{j i} \geq 1$, which means that the ask price is always greater than the bid price. The case where $\pi_{t}^{i j} \pi_{t}^{j i}=1$ corresponds to the situation where the ask and bid prices are the same, i.e. there is no friction.

All over this paper, we shall consider the so-called efficient friction case:

Standing Assumption (EF) : $\pi_{t}^{i j} \pi_{t}^{j i}>1$ for all $i \neq j \leq d$ and $t \in \mathbb{T}$.

It means that ask prices are always strictly greater than bid prices.

As in [8] and [9], we model portfolios as $d$-dimensional processes, each component $i$ corresponding to the number of units of asset $i$ detained. The composition $V_{t}$ of a portfolio holding at time $t$ can be changed by acting on the financial market. If $\xi_{t}$ denotes the net number of additional units of each asset in the portfolio after trading at time $t$, it should satisfy the standard self-financing condition. In our context, this means that $\xi_{t} \in-K_{t}$, since we allow to discard any non-negative number of holdings, where, for each $\omega \in \Omega$,

$$
K_{t}(\omega):=\operatorname{conv}\left(\pi_{t}^{i j}(\omega) e_{i}-e_{j}, e_{i} ; i, j \leq d\right),
$$

where $e_{i}$ stands for the $i$-th unit vector of $\mathbb{R}^{d}$ defined by $e_{i}^{k}=1_{i=k}$.

Note that $V_{t} \in K_{t}$ means that there exists $\xi_{t} \in-K_{t}$ such that $V_{t}+\xi_{t}=0$. This explains why $K_{t}$ is usually referred to as the solvency cone, i.e. the set of positions 
that can be turned into positions with non-negative entries by immediately trading on the market.

As in Bouchard and Pham [1], we also allow for production. In [1], the production regime depends only on the inventories in some production assets. Here, we consider a different approach based on a full control of the production regimes. Namely, we consider a family of random maps $\left(R_{t}\right)_{t \in \mathbb{T}}$ from $\mathbb{R}_{+}^{d}$ into $\mathbb{R}^{d}$ that corresponds to production functions. It turns $\beta_{t}$ units of assets taken from the portfolio at time $t$ into $R_{t+1}\left(\beta_{t}\right)$ additional units of assets in the portfolio at time $t+1$. For the moment, we only assume that $R_{t+1}$ is $\mathcal{F}_{t+1}$ measurable, in the sense that $R_{t+1}(\beta) \in L^{0}\left(\mathbb{R}^{d}, \mathcal{F}_{t+1}\right)$ for all $\beta \in L^{0}\left(\mathbb{R}_{+}^{d}, \mathcal{F}_{t}\right)$. The control $\beta_{t}$ can be associated to a regime of production. Componentwise, the greater $\beta_{t}$ gets, the more the producer is putting into the production system.

All together, a strategy is a pair of adapted processes

$$
(\xi, \beta) \in \mathcal{A}_{0}:=L^{0}\left((-K) \times \mathbb{R}_{+}^{d}, \mathbb{F}\right)
$$

i.e., such that $\left(\xi_{t}, \beta_{t}\right) \in L^{0}\left(\left(-K_{t}\right) \times \mathbb{R}_{+}^{d}, \mathcal{F}_{t}\right)$ for all $0 \leq t \leq T$. The corresponding portfolio process, starting from 0 , can be written as $V^{\xi, \beta}=\left(V_{t}^{\xi, \beta}\right)_{t \in \mathbb{T}}$ where

$$
V_{t}^{\xi, \beta}:=\sum_{s=0}^{t}\left(\xi_{s}-\beta_{s}+R_{s}\left(\beta_{s-1}\right) 1_{s \geq 1}\right)
$$

Example 2.1 Let us consider a market model where the agent produces electricity that can then be sold on the spot market. For ease of presentation, we only consider the case where the production takes place in a single monetary zone, say Euro, but the model can be extended to several currencies. The market consists of three assets: the first one is cash, the second one is coal and the last one is fuel. Allowed self-financed strategies $\xi$ are described by the bid-ask process $\left(\pi^{i j}\right)_{1 \leq i, j \leq 3}$. The agent can use coal or fuel for production purpose, but can also buy a one period ahead delivery contract to small local electricity producers. Given a regime $\beta_{t}$, the producer obtains a return $r_{t+1}^{1}\left(\beta_{t}\right)$ labeled in cash at time $t+1$, depending on the electricity spot price. Since he does not produce coal or fuel, there is no return in these two assets. As a consequence, the production function $R_{t+1}$ has the form $\left(r_{t+1}^{1}, 0,0\right)$, and is a random $\mathcal{F}_{t+1}$-measurable function.

Remark 2.1 Observe that we do not impose constraints on portfolio processes. In particular, one can consume some assets for production purposes although one does not have them. This means that one can borrow some assets to use them in the production 
system. As usual, additional convex constraints could be introduced without much difficulty.

In the following, we shall denote by

$$
A_{t}^{R}(T):=\left\{\sum_{s=t}^{T} \xi_{s}-\beta_{s}+R_{s}\left(\beta_{s-1}\right) 1_{s \geq t+1},(\xi, \beta) \in \mathcal{A}_{0}\right\}, t \leq T,
$$

the set of portfolio holdings that are attainable at time $T$ by trading from time $t$ with a zero initial holding.

Remark 2.2 The sequence of random cones $K=\left(K_{t}\right)_{t \in \mathbb{T}}$ is defined here through the bid-ask process $\pi$. However, it should be clear that all our analysis remains true in a more abstract framework. Namely, one could only consider that $K$ is a sequence of closed convex cones such that $K_{t}$ is $\mathcal{F}_{t}$-measurable, $\mathbb{R}_{+}^{d} \subset K_{t}$ and $K_{t} \cap\left(-K_{t}\right)=\{0\}$ for all $t \leq T$.

\subsection{The no-arbitrage condition}

In a model without production, i.e., $R \equiv 0$, Rásonyi [16] recently proposed to consider the following no-arbitrage of the second kind condition, also called no-sure gain in liquidation value, $\mathbf{N G V}$ in short:

NA2 $^{0}: \quad\left(\zeta+A_{t}^{0}(T)\right) \cap L^{0}\left(K_{T}, \mathcal{F}\right) \neq\{0\} \Rightarrow \zeta \in L^{0}\left(K_{t}, \mathcal{F}\right)$, for all $\zeta \in L^{0}\left(\mathbb{R}^{d}, \mathcal{F}_{t}\right)$ and $t \leq T$.

This means that we cannot end-up at time $T$ with a solvent position without taking any risk if the initial position is not already solvent.

In this paper, we shall impose a similar condition on the pure financial part of the model, i.e., there is no-arbitrage of the second kind for strategies of the form $(\xi, 0) \in \mathcal{A}_{0}$. Contrary to [1], we do not exclude arbitrages coming from production whenever the production regime is small. We only exclude marginal arbitrages for high regimes of production in the following sense:

Definition 2.1 1. Given $L \in L^{0}\left(\mathbb{M}^{d}, \mathbb{F}\right)$, we say that there is no arbitrage of the second kind for the linear production map $L$, in short $\mathbf{N A 2}{ }^{L}$ holds, if

(i) $\zeta-\beta+L_{t+1} \beta \in L^{0}\left(K_{t+1}, \mathcal{F}_{t+1}\right) \Rightarrow \zeta \in K_{t}$,

(ii) $-\beta+L_{t+1} \beta \in L^{0}\left(K_{t+1}, \mathcal{F}_{t+1}\right) \Rightarrow \beta=0$, 
for all $(\zeta, \beta) \in L^{0}\left(\mathbb{R}^{d} \times \mathbb{R}_{+}^{d}, \mathcal{F}_{t}\right)$ and $t<T$.

2. We say that there is no marginal arbitrage of the second kind for high production regimes, in short NMA2 holds, if there exists $(c, L) \in L^{0}\left(\mathbb{R}^{d}, \mathbb{F}\right) \times L^{0}\left(\mathbb{M}^{d}, \mathbb{F}\right)$ such that $\mathbf{N A} 2^{L}$ holds and

$$
c_{t+1}+L_{t+1} \beta-R_{t+1}(\beta) \in L^{0}\left(K_{t+1}, \mathcal{F}_{t+1}\right) \text { for all } \beta \in L^{0}\left(\mathbb{R}_{+}^{d}, \mathcal{F}_{t}\right) \text { and } t<T \text {. }
$$

The condition (2.5) means that the production function $R_{t}$ admits an affine upper bound. In most production models, each component $R_{t}^{i}, i \leq d$, is concave, so that $R_{t}$ typically admits such a bound. In (i) and (ii), we focus on the production model where $R$ is replaced with the linear map associated to $L$. The fact that we consider the production map $\beta \mapsto L_{t+1} \beta$ instead of $\beta \mapsto c_{t+1}+L_{t+1} \beta$ coincides with the idea that we only want to avoid arbitrages for high production regimes: for large values of $\left|L_{t+1} \beta\right|,\left|c_{t+1}\right|$ becomes negligible.

For $L \equiv 0$, the condition (i) is equivalent to the NGV condition of [16], this follows from a simple induction under the standing assumption (EF) above. Our version is a simple extension to the production-investment model. Condition (i) means that, even if we produce, we cannot almost surely have a solvent position at time $t+1$ if the position was not already solvent at time $t$. Condition (ii) means that producing may lead to net losses.

In the following, unless otherwise specified, we shall consider $(c, L)$ as given once for all, and such that (2.5) is satisfied (whenever NMA2 holds). We shall refer to the linear model as the one where $R$ is replaced by $\beta \mapsto L \beta$.

Remark 2.3 If $\operatorname{esssup}\left\{\left|R_{t+1}(\beta)\right|, \beta \in L^{0}\left(\mathbb{R}_{+}^{d}, \mathcal{F}\right)\right\} \in L^{\infty}$ for all $t<T$, then one can choose $L \equiv 0$. In this case, NMA2 coincides with the NGV condition of [16] on the pure financial part, i.e., the no-arbitrage condition is set only on strategies of the form $(\xi, 0)$.

Let us now illustrate the no-arbitrage condition NMA2 in the context of the model described in Example 2.1.

Example 2.2 Consider the electricity production model of Example 2.1. If $r_{t}^{1}$ is $\mathbb{P}-$ a.s. concave and non-decreasing, then $r_{t}^{1}(\alpha \beta) / \alpha$ admits $\mathbb{P}-$ a.s. a limit $L_{t}^{1}(\beta)$ as $\alpha \rightarrow$ $\infty$, where the map $\beta \mapsto L_{t}^{1}(\beta)$ is $\mathbb{P}-$ a.s. linear. It follows that $R_{t}(\alpha \beta) / \alpha$ admits a limit as $\alpha \rightarrow \infty$ with can be associated to a random matrix $L_{t}$ of dimension 3 . Moreover, we clearly can find $c_{t} \in L^{0}\left(\mathbb{R}^{d}, \mathcal{F}_{t}\right)$ such that (2.5) holds. 
We consider now a specific model of such a situation. Recall that $\beta_{t}^{2}$ (resp. $\beta_{t}^{3}$ ) denotes the number of units of coal (resp. fuel) sent to power plants using coal (resp. fuel) at time $t$. Hereafter coal and fuel are called technologies 2 and 3. The agent has $n_{i} \geq 1$ power plants that use the technology $i=2,3$. The $k$-th power plant that uses the technology $i$ has a maximal capacity $\Xi_{t+1}^{i k} \in L^{0}\left(\mathbb{R}_{+} \cup\{\infty\}, \mathcal{F}_{t+1}\right)$ for the time period $[t, t+1], i=2,3$ and $k=1, \ldots, n_{i}$. The case $\Xi_{t+1}^{i k}=\infty$ means that there is no limit on the number of quantities that can be treated. Each of them convert one unit of raw material sent to the plant at time $t$ into $l_{t+1}^{i k} \in L^{0}\left(\mathbb{R}_{+}, \mathcal{F}_{t+1}\right) M$ Wh of energy that are sold on the spot market at a price $s_{t+1} \in L^{0}\left(\mathbb{R}, \mathcal{F}_{t+1}\right)$. The factor $l_{t+1}^{i k}$ is called the heat rate of the $k$-th power plant, which uses the technology $i$. The randomness of $\Xi_{t+1}^{i k}$ and $l_{t+1}^{i k}$ allows one to model possible random outages or temperature effects in the production process, for instance. For ease of presentation, we assume that the producer has an idea which power plant is more efficient and uses that one in priority. Without loss of generality, we can assume that power plants are ordered by efficiency, namely

$$
l_{t+1}^{i k} \geq l_{t+1}^{i(k+1)} \mathbb{P}-\text { a.s. for all } k \in\left[1, n_{i}-1\right], i=2,3 \text { and } t<T .
$$

The production function $r^{1 i}$ associated to the technology $i=2,3$ is thus given by

$$
r_{t+1}^{1 i}\left(\beta^{i}\right)=s_{t+1} \sum_{k=1}^{n_{i}}\left(l_{t+1}^{i k} \min \left\{\beta^{i}-\bar{\Xi}_{t+1}^{i k} ; \Xi_{t+1}^{i k}\right\}^{+}\right)-\sum_{k=1}^{n_{i}} \gamma_{t+1}^{i k} \mathbf{1}_{\left\{\beta^{i} \geq \Xi_{t+1}^{i k}\right\}}
$$

where $\bar{\Xi}_{t+1}^{i k}:=\sum_{1=\ell \leq(k-1)} \Xi_{t+1}^{i \ell}$ denotes the maximal capacity of the best $k-1$ plants, $y^{+}$denotes the positive part of a real number $y$, and $\gamma_{t+1}^{i k} \in L^{0}\left(\mathbb{R}_{+}, \mathcal{F}_{t+1}\right)$ stands for a (possibly random) fixed cost associated to the $k$-th power plant (e.g., a starting cost). We denote by $\beta_{t}^{1}$ the amount of cash used at time $t$ to buy one period ahead delivery contracts from small local electricity producers. The price of these contracts at time $t$ is $f_{t} \in L^{0}\left((0, \infty), \mathcal{F}_{t}\right)$ per $M W h$. Thus, consuming $\beta_{t}^{1}$ units of cash at time $t$ produces

$$
r_{t+1}^{11}\left(\beta_{t}^{1}\right):=\frac{s_{t+1}}{f_{t}} \beta_{t}^{1}
$$

units of cash at time $t+1$, once all $M W h$ have been sold on the spot market at the spot price $s_{t+1}$.

All together, the production map is given by

$$
R_{t+1}\left(\beta_{t}\right)=\left(r_{t+1}^{1}\left(\beta_{t}\right):=\sum_{i=1}^{3} r_{t+1}^{1 i}\left(\beta_{t}^{i}\right), 0,0\right) .
$$


Note that $r_{t+1}^{1}$ is not concave, except if $\gamma^{i k}=0$ for all $i, k$, and $s_{t+1} \geq 0$, which may not be the case on the electricity spot market. However, $R_{t+1}$ satisfies (2.5) with $L$ defined by

$$
L_{t+1}^{11}:=s_{t+1} / f_{t}, L_{t+1}^{1 i}:=\mathbf{1}_{\left\{k_{t}^{i}<\infty\right\}} s_{t+1} l_{t+1}^{i k_{t}^{i}} \text { for } i=2,3 \text {, and } L_{t+1}^{j i}:=0 \text { for } j \neq 1,
$$

where

$$
k_{t}^{i}:=\min \left\{k \leq n_{i}: \Xi_{t+1}^{i k}=\infty\right\},
$$

with the usual convention $\min \emptyset=\infty$. The above choice of $L$ is the smallest possible one (component-by-component) under (2.6). As for the minimal possible c (componentby-component) such that (2.5) holds, it takes the form $c_{t+1}=\left(c_{t+1}^{1}, 0,0\right)$ with

$$
c_{t+1}^{1}=\max _{\beta \in \mathbb{R}_{+}^{3}}\left(r_{t+1}^{1}(\beta)-\sum_{i=1}^{3} L_{t+1}^{1 i} \beta^{i}\right),
$$

which is $\mathbb{P}-$ a.s. finite.

We conclude this section with a remark that highlights the differences between the notion of no marginal arbitrage for high production regimes introduced here and the (seemingly close) notions of no marginal arbitrage and no scalable arbitrage discussed in $[13]$.

Remark 2.4 1. In [13] and the references therein, the author discusses the notion of no marginal arbitrage in the context of discrete time models with stock prices depending in a convex way on the quantity to buy/sell. In the terminology of this paper, a marginal arbitrage has to be understood as an arbitrage obtained when trading the marginal price process associated to infinitesimal trades. In our context, where the non-linearity only comes from the production map $R$, this would (essentially) correspond to an arbitrage obtained for infinitesimal values of $\beta$, i.e. marginally around $\beta=0$. Here, we also consider arbitrages that can happen marginally, but, as explained above, as a "surplus" around large regimes/values of $\beta$ and not around 0 . This explains why we use the terminology of marginal arbitrage for high production regimes. Clearly, the two notions are very different.

2. In [13], the author also discusses the notion of no scalable arbitrage. It expresses the fact that an arbitrage cannot be arbitrarily scaled by a positive scalar. In our setting, the no scalable arbitrage condition would read:

$$
\bigcap_{\alpha>0} \alpha A_{0}^{R}(T) \cap L^{0}\left(\mathbb{R}_{+}^{d}, \mathcal{F}\right)=\{0\}
$$


For real valued concave maps $R$ satisfying $R(0)=0$, the no scalable arbitrage condition (essentially) means that the usual no-arbitrage condition holds when considering the production map $\beta \mapsto \nabla R(\infty) \beta$, whenever we can give a sense to the gradient $\nabla R$ and it admits a limit at infinity. In this case, with $L:=\nabla R(\infty)$ in NMA2, we see that (at least formally) our no marginal arbitrage of second kind condition for high production regimes, could be viewed as a no scalable arbitrage of second kind condition.

This is not the case in general. Apart from technicalities (for instance, we do not assume necessarily concavity, except for the super-hedging theorems of Section 3.1), the main reason is that we are not interested in arbitrages that are scalable but arbitrages that can appear marginally as a "surplus" given that the production regime is already high. To illustrate this, let us consider a very simple (degenerate) two dimensional model with two periods $t=0,1$. We take $\pi_{t}^{12}=2$ and $\pi_{t}^{21}=1$ for $t=0,1, R_{1}^{1}(\beta)=$ $-\bar{c}+\bar{L}_{1} \beta^{1}$ and $R^{2}=0$ where $\bar{c}>0$ is a constant and $\mathbb{P}\left[\bar{L}_{1}=1\right]=0$. This model satisfies $(2.5)$ with $c_{1}=(-\bar{c}, 0), L_{1}^{11}=\bar{L}_{1}, L_{1}^{i j}=0$ for $(i, j) \neq(1,1)$. In this model, direct computations show that a claim of the form $g=\left(\lambda_{g}\left(\bar{L}_{1}-1\right), 0\right)$, with $\lambda_{g}>0$, is scalable, i.e., belongs to $\cap_{\alpha>0} \alpha A_{0}^{R}(T)$, if and only if, for each $\alpha>0$, one can find $\beta_{0}^{1 \alpha} \in \mathbb{R}_{+}$and $\gamma^{\alpha} \in L^{0}\left(\mathbb{R}_{+}, \mathcal{F}_{1}\right)$ such that $\beta_{0}^{1 \alpha}=\lambda_{g} / \alpha+\left(\bar{c}+\gamma^{\alpha}\right) /\left(\bar{L}_{1}-1\right)$. Because $\bar{c}>0$ and $\gamma^{\alpha}$ has to take non-negative values, this is not possible, except in the case where $\bar{L}_{1}$ is not random (otherwise $\beta_{0}^{1, \alpha}$ would be a random variable as opposed to a real number). This shows that such claims are not scalable (in general) in the sense that they do not belong to $\cap_{\alpha>0} \alpha A_{0}^{R}(T)$. Hence, in general, the no scalable arbitrage condition does not say anything on such claims, while our NMA2 condition says exactly that they cannot belong to $L^{0}\left(\mathbb{R}_{+}^{2}, \mathcal{F}_{1}\right) \backslash\{0\}$.

\subsection{Dual characterization of the no-arbitrage condition and closedness properties}

Before we state our main results, let us introduce some additional notations and definitions.

We first define the positive dual cone process $K^{*}=\left(K_{t}^{*}\right)_{t \in \mathbb{T}}$ associated to $K$ by

$$
K_{t}^{*}(\omega):=\left\{z \in \mathbb{R}^{d}: x^{\prime} z \geq 0 \text { for all } x \in K_{t}(\omega)\right\}, \omega \in \Omega .
$$

For $t \leq \tau \leq T$, we denote by $\mathcal{M}_{t}^{\tau}\left(\operatorname{int} K^{*}\right)$ the set of martingales $Z$ with positive components satisfying $Z_{s} \in L^{0}\left(\operatorname{int} K_{s}^{*}, \mathcal{F}_{s}\right)$ for all $t \leq s \leq \tau$.

Elements of $\mathcal{M}_{t}^{T}\left(\operatorname{int} K^{*}\right)$ are called strictly consistent price systems, on $[t, T]$, in [17]. In a fictitious market without transaction costs admitting a martingale measure, $Z$ 
could be interpreted in a way such that the relative prices evolve in the interior of the corresponding bid-ask intervals of the original model induced by $\pi$, i.e. are more favorable for the financial agent. Indeed, one easily checks that

$$
K_{t}^{*}(\omega):=\left\{z \in \mathbb{R}_{+}^{d}: z^{j} \leq z^{i} \pi_{t}^{i j}(\omega) \text { for all } i \neq j \leq d\right\}
$$

Taking the first asset as a numéraire, i.e., for $\bar{Z}$ defined as $\bar{Z}_{s}^{i}:=Z_{s}^{i} / Z_{s}^{1}$ for $t \leq s \leq$ $T$ and for any $Z \in \mathcal{M}_{t}^{T}\left(\operatorname{int} K^{*}\right)$, it follows that $\bar{Z}$ is a martingale on $[t, T]$ under the measure $\mathbb{Q}$ induced by the conditional density process $\left(Z_{s}^{1} / Z_{t}^{1}\right)_{t \leq s \leq T}$ and satisfies $\bar{Z}_{s}^{j} / \bar{Z}_{s}^{i}<\pi_{s}^{i j}$ for $t \leq s \leq T$.

Remark 2.5 Note that the condition (EF) above is actually equivalent to $\operatorname{int} K_{t}^{*} \neq \emptyset$ for all $t \leq T$. This follows from (2.8).

All together, elements of $\mathcal{M}_{0}^{T}\left(\operatorname{int} K^{*}\right)$ play a similar role as equivalent martingale measures in frictionless markets, see [17] and the references therein. In particular, it was shown in [16] that, for $L \equiv 0$, the no-arbitrage condition $\mathbf{N A 2} \mathbf{2}^{0}$ is equivalent to:

$\mathbf{P C E}^{0}$ : for each $0 \leq t \leq T$ and $X \in L^{1}\left(\operatorname{int} K_{t}^{*}, \mathcal{F}_{t}\right)$, there exists a process $Z \in$ $\mathcal{M}_{t}^{T}\left(\operatorname{int} K^{*}\right)$ satisfying $Z_{t}=X$.

This not only means that the no-arbitrage condition $\mathbf{N A} 2^{0}$ implies the existence of a strictly consistent price system, but that strictly consistent price systems defined on any subinterval $[t, \tau]$ can also be extended consistently on $[t, T]$. Indeed for $Z \in$ $\mathcal{M}_{t}^{\tau}\left(\operatorname{int} K^{*}\right)$, one can find a strictly consistent price system $\tilde{Z} \in \mathcal{M}_{t}^{T}\left(\operatorname{int} K^{*}\right)$ such that $\tilde{Z}=Z$ on $[t, \tau]$.

Such a property is obvious in frictionless markets but in general not true in our multivariate setting where the geometry of the cones $\left(K_{t}^{*}\right)_{t \in \mathbb{T}}$ is non-trivial.

In our production-investment setting, such price systems should also take the production function into account. When it is linear and given by the random matrix process $L$, the cost in units at time $t$ of a return (in units) $L_{t+1} \beta$ at time $t+1$ is $\beta \in L^{0}\left(\mathbb{R}_{+}^{d}, \mathcal{F}_{t}\right.$ ). Otherwise stated, one can build the position $\left(L_{t+1}-I_{d}\right) \beta$ at time $t+1$ from a zero holding at time $t$. For the price system $\bar{Z}$ and the associated pricing measure $\mathbb{Q}$, see the discussion above, the value at time $t$ of this return is $\mathbb{E}^{\mathbb{Q}}\left[\bar{Z}_{t+1}^{\prime}\left(L_{t+1}-I_{d}\right) \beta \mid \mathcal{F}_{t}\right]$. If the fictitious price system is strictly more favorable than the original one, one should actually be able to choose it in such a way that $\mathbb{E}^{\mathbb{Q}}\left[\bar{Z}_{t+1}^{\prime}\left(L_{t+1}-I_{d}\right) \beta \mid \mathcal{F}_{t}\right]<0$ for all $\beta \in L^{0}\left(\mathbb{R}_{+}^{d}, \mathcal{F}_{t}\right) \backslash\{0\}$. 
The above discussion leads to the introduction of the set $\mathcal{L}_{t}^{\tau}\left(\operatorname{int} \mathbb{R}_{-}^{d}\right)$ of martingales $Z$ on $[t, \tau]$ with positive components satisfying $\mathbb{E}\left[\left|Z_{s+1}^{\prime}\left(L_{s+1}-I_{d}\right)\right| \mid \mathcal{F}_{s}\right]<\infty$ as well as $\mathbb{E}\left[Z_{s+1}^{\prime}\left(L_{s+1}-I_{d}\right) \mid \mathcal{F}_{s}\right] \in \operatorname{int} \mathbb{R}_{-}^{d}$ for all $t \leq s<\tau, t<\tau \leq T \mathbb{P}-$ a.s.

Our first main result extends the property $\mathbf{N A} 2^{0} \Leftrightarrow \mathbf{P C E}^{0}$ to $\mathbf{N A 2}{ }^{L} \Leftrightarrow \mathbf{P C E}^{L}$ where $\mathbf{P C E}^{L}$ : for each $0 \leq t \leq T$ and $X \in L^{1}\left(\operatorname{int} K_{t}^{*}, \mathcal{F}_{t}\right)$, there exists a process $Z \in$ $\mathcal{M}_{t}^{T}\left(\operatorname{int} K^{*}\right) \cap \mathcal{L}_{t}^{T}\left(\operatorname{int} \mathbb{R}_{-}^{d}\right)$ satisfying $Z_{t}=X$.

\section{Theorem 2.1 $\mathrm{NA2}^{L} \Leftrightarrow \mathrm{PCE}^{L}$.}

Remark 2.6 Note that the property $\mathbf{P C E}^{L}$ allows one to construct (in theory) all the elements of $\mathcal{M}_{0}^{T}\left(\operatorname{int} K^{*}\right) \cap \mathcal{L}_{0}^{T}\left(\operatorname{int} \mathbb{R}_{-}^{d}\right)$ by a simple forward induction. First, one can start with any $Z_{0} \in \operatorname{int} K_{0}^{*}$. Assuming that a given $Z \in \mathcal{M}_{0}^{t}\left(\operatorname{int} K^{*}\right) \cap \mathcal{L}_{0}^{t}\left(\operatorname{int} \mathbb{R}_{-}^{d}\right)$ has been constructed, one can then choose any random variable $Z_{t+1} \in L^{0}\left(\operatorname{int} K_{t+1}^{*}, \mathcal{F}_{t+1}\right)$ such that $\mathbb{E}\left[Z_{t+1} \mid \mathcal{F}_{t}\right]=Z_{t}$ and $\mathbb{E}\left[Z_{t+1}^{\prime}\left(L_{t+1}-I_{d}\right) \mid \mathcal{F}_{t}\right] \in \operatorname{int} \mathbb{R}_{-}^{d}$. These correspond to simple linear inequalities. When $\Omega$ is finite, the set of such random variables can be described explicitly.

By similar arguments as developed in Lemma 3.2 in [2], the existence of $Z \in \mathcal{M}_{0}^{T}\left(\operatorname{int} K^{*}\right) \cap$ $\mathcal{L}_{0}^{T}\left(\operatorname{int} \mathbb{R}_{-}^{d}\right)$ then allows one to provide a $L^{1}$ upper bound on strategies $(\xi, \beta) \in \mathcal{A}_{0}$ satisfying $V_{T}^{\xi, \beta}+\kappa \in K_{T}$ for some $\kappa \in \mathbb{R}^{d}$. However, because no integrability condition is imposed a-priori on $c$, we require the additional assumption:

$$
\exists \check{Z} \in \mathcal{M}_{0}^{T}\left(\operatorname{int} K^{*}\right) \cap \mathcal{L}_{0}^{T}\left(\operatorname{int} \mathbb{R}_{-}^{d}\right) \text { s.t. } \mathbb{E}\left[\left|\check{Z}_{T}^{\prime} c_{t}\right|\right]<\infty \forall 0<t \leq T .
$$

Lemma 2.1 Assume that (2.9) holds. Then, there exists $\mathbb{Q} \sim \mathbb{P}$ and a constant $\alpha \geq 0$, such that, for all $\kappa \in \mathbb{R}^{d}$ and $(\xi, \beta) \in \mathcal{A}_{0}$ satisfying $V_{T}^{\xi, \beta}+\kappa \in K_{T}$, one has:

$$
\mathbb{E}^{\mathbb{Q}}\left[\sum_{0 \leq t \leq T}\left(\left|\xi_{t}\right|+\left|\beta_{t}\right|\right)\right] \leq \alpha\left(\mathbb{E}\left[\check{Z}_{T} C_{0}^{T}\right]+\check{Z}_{0}^{\prime} \kappa\right)
$$

where

$$
C_{t}^{T}:=\sum_{s=t+1}^{T} c_{s}, t<T .
$$

Remark 2.7 Given $(\xi, \beta) \in \mathcal{A}_{0}$, let us define

$$
\mathcal{V}_{t}^{\xi, \beta}:=\sum_{s=0}^{t}\left(\xi_{s}-\beta_{s}+L_{s} \beta_{s-1} 1_{s \geq 1}\right) .
$$


In view of Theorem 2.1, applying Lemma 2.1 to the case $R(\beta)=0+L \beta$, i.e. $c=0$, leads to the following corollary: Assume that $\mathbf{N A 2}^{L}$ holds. Then, there exists $\mathbb{Q} \sim \mathbb{P}$, $Z_{0} \in \operatorname{int} K_{0}^{*}$ and a constant $\alpha \geq 0$ such that, for all $\kappa \in \mathbb{R}^{d}$ and $(\xi, \beta) \in \mathcal{A}_{0}$ satisfying $\mathcal{V}_{T}^{\xi, \beta}+\kappa \in K_{T}$, one has:

$$
\mathbb{E}^{\mathbb{Q}}\left[\sum_{0 \leq t \leq T}\left(\left|\xi_{t}\right|+\left|\beta_{t}\right|\right)\right] \leq \alpha Z_{0}^{\prime} \kappa
$$

The last remark combined with Komlos Lemma readily implies that the sets

$$
A_{t}^{L}(T):=\left\{\sum_{s=t}^{T}\left(\xi_{s}-\beta_{s}+L_{s}\left(\beta_{s-1}\right) 1_{s \geq t+1}\right),(\xi, \beta) \in \mathcal{A}_{0}\right\},
$$

are Fatou-closed, in the sense that the limit in probability of sequences of elements $\left(g_{n}\right)_{n \geq 1} \subset A_{t}^{L}(T)$ satisfying $g_{n}+\kappa \in K_{T}$ for all $n \geq 1$ belongs to $A_{t}^{L}(T)$ as well. Under (2.9), a similar result could be easily proved by appealing to Lemma 2.1 for the sets $A_{t}^{R}(T)$, recall (2.4), under the following upper-semicontinuity assumption:

$$
\text { (USC) : } \limsup _{\beta \in \mathbb{R}_{+}^{d}, \beta \rightarrow \beta^{0}} R_{t}(\beta)-R_{t}\left(\beta^{0}\right) \in-K_{t} \text { for all } \beta^{0} \in \mathbb{R}_{+}^{d}, t \leq T
$$

where the limsup is taken component-by-component. Such Fatou-closedness properties are sufficient for applications, but they require (2.9). In order to deal with the general case, i.e., when (2.9) may not hold, we need to use more elaborate arguments, which actually allows one to obtain the following stronger closedness property.

Theorem 2.2 $A_{0}^{L}(T)$ is closed in probability under $\mathbf{N A 2}{ }^{L}$. The same holds for $A_{0}^{R}(T)$ under NMA2 and (USC).

Example 2.3 We continue the study of Example 2.2. Hereafter, we assume that conditions (2.1) and (EF) are satisfied. Note that the condition (ii) of $\mathbf{N A 2}^{L}$ is satisfied if and only if, for all $t \leq T-1$ and $\beta_{t} \in L^{0}\left(\mathbb{R}_{+}^{3}, \mathcal{F}_{t}\right)$,

$$
\sum_{i=1}^{3}\left(L_{t+1}^{1 i}-\pi_{t+1}^{1 i}\right) \beta_{t}^{i} \geq 0 \Rightarrow \beta_{t}=0
$$

which is equivalent to

$$
\mathbb{P}\left[s_{t+1}<f_{t} \mid \mathcal{F}_{t}\right]>0 \text { and } \mathbb{P}\left[\mathbf{1}_{\left\{k_{t}^{i}<\infty\right\}} s_{t+1} l_{t+1}^{i k_{t}^{i}}<\pi_{t+1}^{1 i} \mid \mathcal{F}_{t}\right]>0 \text { for } i=2,3 .
$$

Assuming that the above condition is satisfied, then (i) of $\mathbf{N A 2}{ }^{L}$ is equivalent to the existence of an element $Z \in \mathcal{M}_{0}^{T}\left(\operatorname{int} K^{*}\right) \cap \mathcal{L}_{0}^{T}\left(\operatorname{int} \mathbb{R}_{-}^{d}\right)$. Let $\mathbb{Q} \sim \mathbb{P}$ be defined by $d \mathbb{Q} / d \mathbb{P}=$ 
$Z_{T}^{1}$ and $\bar{Z}:=Z / Z^{1}$. As in [8], [17] and [16], the fact that $Z \in \mathcal{M}_{0}^{T}\left(\operatorname{int} K^{*}\right)$ is equivalent to $\bar{Z}^{i} / \bar{Z}^{j}<\pi^{j i}$ for all $i \neq j$, and each $\bar{Z}^{i}$ is a $\mathbb{Q}$-martingale, $i=2,3$. The new condition $Z \in \mathcal{L}_{0}^{T}\left(\operatorname{int} \mathbb{R}_{-}^{d}\right)$ is equivalent to $\mathbb{E}^{\mathbb{Q}}\left[s_{t+1} \mid \mathcal{F}_{t}\right]<f_{t}$ and $\mathbb{E}^{\mathbb{Q}}\left[\mathbf{1}_{\left\{k_{t}^{i}<\infty\right\}} s_{t+1} l_{t+1}^{i k_{t}^{i}}-\bar{Z}_{t+1}^{i} \mid \mathcal{F}_{t}\right]$ $=\mathbb{E}^{\mathbb{Q}}\left[\mathbf{1}_{\left\{k_{t}^{i}<\infty\right\}} s_{t+1} l_{t+1}^{i k_{t}^{i}} \mid \mathcal{F}_{t}\right]-\bar{Z}_{t}^{i}<0$ for $i=2,3$.

Note that (USC) trivially holds in this example, so that Theorem 2.2 implies that $A_{0}^{R}(T)$ is closed in probability whenever the above conditions are satisfied.

\section{Applications}

\subsection{Super-hedging theorems}

As usual, the closedness property allows one to derive dual formulations for the set of attainable claims. We first formulate it in the linear model. In this section, we denote by $\mathcal{M}_{0}^{T}\left(K^{*}\right)$ the set of martingales $Z$ satisfying $Z_{s} \in L^{0}\left(K_{s}^{*}, \mathcal{F}_{s}\right)$ for all $s \leq T$, and by $\mathcal{L}_{0}^{T}\left(\mathbb{R}_{-}^{d}\right)$ the set of martingales $Z$ with non-negative components satisfying $\mathbb{E}\left[\left|Z_{s+1}^{\prime}\left(L_{s+1}-I_{d}\right)\right| \mid \mathcal{F}_{s}\right]<\infty$ and $\mathbb{E}\left[Z_{s+1}^{\prime}\left(L_{s+1}-I_{d}\right) \mid \mathcal{F}_{s}\right] \in \mathbb{R}_{-}^{d}$ for all $s<T$.

Proposition 3.1 Assume that $\mathbf{N A 2}^{L}$ holds and let $V \in L^{0}\left(\mathbb{R}^{d}, \mathcal{F}\right)$ be such that $V+$ $\kappa \in L^{0}\left(K_{T}, \mathcal{F}\right)$ for some $\kappa \in \mathbb{R}^{d}$. Then the following assertions are equivalent:

(i) $V \in A_{0}^{L}(T)$,

(ii) $\mathbb{E}\left[Z_{T}^{\prime} V\right] \leq 0$ for all $Z \in \mathcal{M}_{0}^{T}\left(K^{*}\right) \cap \mathcal{L}_{0}^{T}\left(\mathbb{R}_{-}^{d}\right)$,

(iii) $\mathbb{E}\left[Z_{T}^{\prime} V\right] \leq 0$ for all $Z \in \mathcal{M}_{0}^{T}\left(\operatorname{int} K^{*}\right) \cap \mathcal{L}_{0}^{T}\left(\operatorname{int} \mathbb{R}_{-}^{d}\right)$.

In the original non-linear model, an abstract dual formulation is also available. However, due to the non-linearity of the set of attainable terminal claims, it requires the introduction of the following support function:

$$
\alpha^{R}(Z):=\sup \left\{\mathbb{E}\left[Z_{T}^{\prime} V\right], V \in A_{0 b}^{R}(T)\right\}, Z \in \mathcal{M}_{0}^{T}\left(K^{*}\right),
$$

where

$$
A_{0 b}^{R}(T):=\left\{V \in A_{0}^{R}(T) \text { s.t. } V+\kappa \in K_{T} \text { for some } \kappa \in \mathbb{R}^{d}\right\} .
$$

Remark 3.1 1. It will be clear from the proof in Section 4.2, see (4.6) with $\varepsilon=0$, that $\alpha^{R}(Z) \leq \mathbb{E}\left[Z_{T}^{\prime} C_{0}^{T}\right]$ for all $Z \in \mathcal{M}_{0}^{T}\left(K^{*}\right) \cap \mathcal{L}_{0}^{T}\left(\mathbb{R}_{-}^{d}\right)$, whenever the last term is well defined, which is in particular the case if $c_{t}$ is essentially bounded from below, component-by-component, for each $t \leq T$.

2. Let $\alpha^{L}$ be defined as $\alpha^{R}$ in the case $R(\beta)=0+L \beta$. Since $0 \in A_{0}^{L}(T)$, we have $\alpha^{L} \geq 0$. On the other hand, 1 . applied to $R(\beta)=0+L \beta$, i.e. $c=0$, implies that $\alpha^{L}(Z) \leq 0$ for all $Z \in \mathcal{M}_{0}^{T}\left(K^{*}\right) \cap \mathcal{L}_{0}^{T}\left(\mathbb{R}_{-}^{d}\right)$. Hence, $\alpha^{L}(Z)=0$ for all $Z \in \mathcal{M}_{0}^{T}\left(K^{*}\right) \cap \mathcal{L}_{0}^{T}\left(\mathbb{R}_{-}^{d}\right)$. 
Moreover, as usual, we shall need the set $A_{0}^{R}(T)$ to be convex, which is easily checked under the additional assumption $(\mathbf{R})(\mathrm{a})$ below. We will also require that bounded strategies lead to $L^{1}$-bounded from below terminal wealth values. We therefore impose the following conditions:

$$
\begin{array}{r}
\text { (a) } \quad \alpha R_{t}\left(\beta_{1}\right)+(1-\alpha) R_{t}\left(\beta_{2}\right)-R_{t}\left(\alpha \beta_{1}+(1-\alpha) \beta_{2}\right) \in-K_{t} \\
\text { for all } \alpha \in L^{0}([0,1], \mathcal{F}), \beta_{1}, \beta_{2} \in L^{0}\left(\mathbb{R}_{+}^{d}, \mathcal{F}\right), t \leq T . \\
\text { (b) } \quad R_{t}^{-}(\beta) \in L^{1}\left(\mathbb{R}^{d}, \mathcal{F}\right) \text { for all } t \leq T \text { and } \beta \in L^{\infty}\left(\mathbb{R}_{+}^{d}, \mathcal{F}\right)
\end{array}
$$

where we have used the notation $R^{-}:=\left(\max \left\{-R^{i}, 0\right\}\right)_{i \leq d}$.

Remark 3.2 The technical condition $(\mathbf{R})(\mathrm{b})$ is by no means restrictive. One can for instance obtain it, whenever there exists a deterministic map $\psi: \mathbb{R}_{+}^{d} \mapsto[1, \infty)$ such that $\operatorname{esssup}\left\{\left|R_{t}^{-}(\beta)\right| / \psi(\beta), t \leq T, \beta \in \mathbb{R}_{+}^{d}\right\}=: \eta \in L^{0}\left(\mathbb{R}_{+}, \mathcal{F}\right)$. Indeed, in this case, it suffices to replace the original probability measure $\mathbb{P}$ by $\tilde{\mathbb{P}} \sim \mathbb{P}$ defined by $d \tilde{\mathbb{P}} / d \mathbb{P}=e^{-\eta} / \mathbb{E}\left[e^{-\eta}\right]$. Since $\tilde{\mathbb{P}} \sim \mathbb{P}$, this does not affect the conditions NA2 ${ }^{L}$, (USC) and $(\mathbf{R})(\mathrm{a})$.

Proposition 3.2 Assume that NMA2, (USC) and (R) hold. Fix $V \in L^{0}\left(\mathbb{R}^{d}, \mathcal{F}\right)$ such that $V+\kappa \in L^{0}\left(K_{T}, \mathcal{F}\right)$, for some $\kappa \in \mathbb{R}^{d}$, and consider the following assertions:

(i) $V \in A_{0}^{R}(T)$,

(ii) $\mathbb{E}\left[Z_{T}^{\prime} V\right] \leq \alpha^{R}(Z)$ for all $Z \in \mathcal{M}_{0}^{T}\left(K^{*}\right)$,

(iii) $\mathbb{E}\left[Z_{T}^{\prime} V\right] \leq \alpha^{R}(Z)$ for all $Z \in \mathcal{M}_{0}^{T}\left(\operatorname{int} K^{*}\right)$.

Then, (i) $\Leftrightarrow(\mathrm{ii}) \Rightarrow$ (iii). If moreover there exists some $Z \in \mathcal{M}_{0}^{T}\left(\operatorname{int} K^{*}\right)$ such that $\alpha^{R}(Z)<\infty$, then (iii) $\Rightarrow$ (ii).

In the case where the linear map $L$ coincides with the asymptotic behavior of $R$, i.e.,

$$
(\mathbf{R L}): \lim _{\eta \rightarrow \infty} R_{t}(\eta \beta) / \eta=L_{t} \beta \quad \text { for all } \beta \in \mathbb{R}_{+}^{d}, t \leq T,
$$

one can restrict to elements in $\mathcal{L}_{0}^{T}\left(\mathbb{R}_{-}^{d}\right)$ (resp. $\mathcal{L}_{0}^{T}\left(\operatorname{int} \mathbb{R}_{-}^{d}\right)$ ) in the above dual formulations.

Proposition 3.3 Let the conditions of Proposition 3.2 hold. Assume further that (RL) is satisfied. Fix $V \in L^{0}\left(\mathbb{R}^{d}, \mathcal{F}\right)$ such that $V+\kappa \in L^{0}\left(K_{T}, \mathcal{F}\right)$, for some $\kappa \in \mathbb{R}^{d}$, and consider the following assertions:

(i) $V \in A_{0}^{R}(T)$,

(ii) $\mathbb{E}\left[Z_{T}^{\prime} V\right] \leq \alpha^{R}(Z)$ for all $Z \in \mathcal{M}_{0}^{T}\left(K^{*}\right) \cap \mathcal{L}_{0}^{T}\left(\mathbb{R}_{-}^{d}\right)$, 
(iii) $\mathbb{E}\left[Z_{T}^{\prime} V\right] \leq \alpha^{R}(Z)$ for all $Z \in \mathcal{M}_{0}^{T}\left(\operatorname{int} K^{*}\right) \cap \mathcal{L}_{0}^{T}\left(\operatorname{int} \mathbb{R}_{-}^{d}\right)$.

Then, (i) $\Leftrightarrow$ (ii) $\Rightarrow$ (iii). If moreover there exists some $Z \in \mathcal{M}_{0}^{T}\left(\operatorname{int} K^{*}\right) \cap \mathcal{L}_{0}^{T}\left(\operatorname{int} \mathbb{R}_{-}^{d}\right)$ such that $\alpha^{R}(Z)<\infty$, then (iii) $\Rightarrow$ (ii).

Remark 3.3 It follows from Remark 3.1 that (i) $\Leftrightarrow$ (ii) $\Leftrightarrow$ (iii) in Propositions 3.2 and 3.3 whenever assumption (2.9) holds. It is the case under NMA2 whenever $c$ is essentially bounded.

\subsection{Utility maximization}

In order to avoid technical difficulties, we shall only discuss here the case of a (possibly) random utility function defined on $\mathbb{R}^{d}$ that is essentially bounded from above. More general cases could be discussed by following the line of arguments of [1].

We therefore let $U$ be a $\mathbb{P}$ - a.s.-upper semi-continuous concave random map from $\mathbb{R}^{d}$ to $[-\infty, 1]$ such that $U(V)=-\infty$ on $\left\{V \notin K_{T}\right\}$ for $V \in L^{0}\left(\mathbb{R}^{d}, \mathcal{F}\right)$. Given an initial holding $x_{0} \in \mathbb{R}^{d}$, we assume that

$$
\mathcal{U}\left(x_{0}\right):=\left\{V \in A_{0}^{R}(T): \mathbb{E}\left[\left|U\left(x_{0}+V\right)\right|\right]<\infty\right\} \neq \emptyset .
$$

Then, existence holds for the associated expected utility maximization problem whenever (USC), (R) and NMA2 hold, and there exists $Z \in \mathcal{M}_{0}^{T}\left(\operatorname{int} K^{*}\right)$ such that $\alpha^{R}(Z)<\infty$. The latter being a consequence of NMA2 when $c$ is essentially bounded (recall Remark 3.1 and Theorem 2.1).

Proposition 3.4 Assume that (USC), (R) and NMA2 hold, and that $\alpha^{R}(Z)<\infty$ for some $Z \in \mathcal{M}_{0}^{T}\left(\operatorname{int} K^{*}\right)$. Assume further that $\mathcal{U}\left(x_{0}\right) \neq \emptyset$. Then, there exists $V\left(x_{0}\right) \in A_{0}^{R}(T)$ such that

$$
\mathbb{E}\left[U\left(x_{0}+V\left(x_{0}\right)\right)\right]=\sup _{V \in \mathcal{U}\left(x_{0}\right)} \mathbb{E}\left[U\left(x_{0}+V\right)\right]
$$

\section{Proofs}

\subsection{No-arbitrage of the second kind in the linear model and $(K, L)$-strictly consistent price systems}

In this section, we first prove that the no-arbitrage of the second kind assumption $\mathbf{N A 2}^{L}$ implies the existence of an element $Z \in \mathcal{M}_{0}^{T}\left(\operatorname{int} K^{*}\right) \cap \mathcal{L}_{0}^{T}\left(\operatorname{int} \mathbb{R}_{-}^{d}\right)$ that we call $(K, L)$-strictly consistent price system. 
The arguments used in the proof of Proposition 4.1 below are inspired by [16], up to non-trivial modifications. This proposition readily implies that $\mathbf{N A 2}{ }^{L} \Rightarrow \mathbf{P C E}^{L}$ up to an obvious induction argument. Before we state it, we recall the following technical result that will be used in our proof, see Lemma 4.3 in [15]. In the following, $B_{1}$ denotes the unit ball of $\mathbb{R}^{d}$.

Lemma 4.1 Let $\mathcal{G} \subset \mathcal{H} \subset \mathcal{F}$ be $\sigma$-algebras. Let $C \subset B_{1}$ be a $\mathcal{H}$-measurable random convex compact set. Then, there exists a $\mathcal{G}$-measurable random convex compact set $\mathbb{E}[C \mid \mathcal{G}] \subset B_{1}$ satisfying

$$
L^{0}(\mathbb{E}[C \mid \mathcal{G}], \mathcal{G})=\left\{\mathbb{E}[\vartheta \mid \mathcal{G}]: \vartheta \in L^{0}(C, \mathcal{H})\right\} .
$$

Proposition 4.1 Assume that NA2 ${ }^{L}$ holds. Then, for all $<T$ and $X \in L^{1}\left(\operatorname{int} K_{t}^{*}, \mathcal{F}_{t}\right)$, there exists $Z \in L^{1}\left(\operatorname{int} K_{t+1}^{*}, \mathcal{F}_{t+1}\right)$ such that $X=\mathbb{E}\left[Z \mid \mathcal{F}_{t}\right], \mathbb{E}\left[\left|Z^{\prime}\left(L_{t+1}-I_{d}\right)\right| \mid \mathcal{F}_{t}\right]<$ $\infty$ and $\mathbb{E}\left[Z^{\prime}\left(L_{t+1}-I_{d}\right) \mid \mathcal{F}_{t}\right] \in \operatorname{int} \mathbb{R}_{-}^{d}$.

Proof We fix $t<T$. For ease of notation, we set $M_{t+1}:=L_{t+1}-I_{d}$. We next define $\gamma_{t+1}:=e^{-\sum_{i, j \leq d}\left|M_{t+1}^{i j}\right|}$ and $\bar{M}_{t+1}:=\gamma_{t+1} M_{t+1}$. Clearly, $\bar{M}_{t+1}$ is essentially bounded.

1. We first show that int $\mathbb{R}_{-}^{d} \subset \operatorname{cone}\left(\operatorname{int} \mathbb{E}\left[\Theta \mid \mathcal{F}_{t}\right]\right)=: H$, where

$$
\Theta:=\left\{\bar{M}_{t+1}^{\prime} y+r,(y, r) \in\left(K_{t+1}^{*} \cap B_{1}\right) \times[0,1]^{d}\right\},
$$

recall that $B_{1}$ is the unit ball of $\mathbb{R}^{d}$. For later use, observe that, since $\bar{M}_{t+1}$ is essentially bounded, Lemma 4.1 applies to $\Theta$ up to an obvious scaling argument.

If int $\mathbb{R}_{-}^{d} \not \subset H$, then $\mathbb{R}_{-}^{d} \not \subset \bar{H}$ on a set $A \in \mathcal{F}_{t}$ with $\mathbb{P}[A]>0$. For each $\omega \in A, \bar{H}(\omega)$ being a closed convex cone, we can then find $p(\omega) \in \mathbb{R}_{-}^{d}$ and $\beta(\omega) \in \mathbb{R}^{d}$ such that

$$
p(\omega)^{\prime} \beta(\omega)<0 \leq q^{\prime} \beta(\omega) \text { for all } q \in \bar{H}(\omega) \text { for } \omega \in A .
$$

By a standard measurable selection argument, see e.g. [3, III-45], one can assume that $p$ and $\beta$ are $\mathcal{F}_{t}$-measurable. The right-hand side of (4.1), Lemma 4.1 and the fact that $K_{t+1}^{*}$ is a cone then imply that

$$
\left(Y^{\prime} \bar{M}_{t+1}+\rho^{\prime}\right) \beta \mathbf{1}_{A} \geq 0 \text { for all }(Y, \rho) \in L^{\infty}\left(K_{t+1}^{*} \times \mathbb{R}_{+}^{d}, \mathcal{F}_{t+1}\right),
$$

which leads to $\beta \mathbf{1}_{A} \in \mathbb{R}_{+}^{d}$ and $\bar{M}_{t+1} \beta \mathbf{1}_{A} \in K_{t+1}$. Since $K_{t+1}$ is a cone, the latter implies $M_{t+1} \beta \mathbf{1}_{A} \in K_{t+1}$. In view of $\mathbf{N A 2}{ }^{L}$, this implies that $\beta \mathbf{1}_{A}=0$, which contradicts the left-hand side of (4.1).

2. We next show that there exists $\tilde{Y} \in L^{\infty}\left(\operatorname{int} K_{t+1}^{*}, \mathcal{F}_{t+1}\right)$ such that $\mathbb{E}\left[\bar{M}_{t+1}^{\prime} \tilde{Y} \mid \mathcal{F}_{t}\right] \in$ int $\mathbb{R}_{-}^{d}$. 
To see this, fix $\eta \in L^{\infty}\left(\operatorname{int} \mathbb{R}_{-}^{d}, \mathcal{F}_{t}\right)$ and $Z \in L^{\infty}\left(\operatorname{int} K_{t+1}^{*}, \mathcal{F}_{t+1}\right)$. Set $\bar{Z}:=\mathbb{E}\left[\bar{M}_{t+1}^{\prime} Z \mid \mathcal{F}_{t}\right]$. We can then find $\varepsilon \in L^{\infty}\left((0,1], \mathcal{F}_{t}\right)$ such that $\eta-\varepsilon \bar{Z} \in L^{\infty}\left(\operatorname{int} \mathbb{R}_{-}^{d}, \mathcal{F}_{t}\right)$. In view of step 1 and Lemma 4.1, there exists $(Y, \rho) \in L^{\infty}\left(K_{t+1}^{*} \times \mathbb{R}_{+}^{d}, \mathcal{F}_{t+1}\right)$ and $\alpha \in L^{0}\left(\operatorname{int} \mathbb{R}_{+}, \mathcal{F}_{t}\right)$ such that $\eta-\varepsilon \bar{Z}=\alpha \mathbb{E}\left[\bar{M}_{t+1}^{\prime} Y+\rho \mid \mathcal{F}_{t}\right]$ or, equivalently, $\eta-\alpha \mathbb{E}\left[\rho \mid \mathcal{F}_{t}\right]=\mathbb{E}\left[\bar{M}_{t+1}^{\prime}(\alpha Y+\varepsilon Z) \mid \mathcal{F}_{t}\right]$. Clearly, $\eta-\alpha \mathbb{E}\left[\rho \mid \mathcal{F}_{t}\right] \in \operatorname{int} \mathbb{R}_{-}^{d}$ and $\alpha Y+\varepsilon Z \in L^{0}\left(\operatorname{int} K_{t+1}^{*}, \mathcal{F}_{t+1}\right)$. The required result is thus obtained for $\tilde{Y}:=(\alpha Y+\varepsilon Z) /(1+\alpha)$.

3. We now show that $\operatorname{int}\left(K_{t}^{*} \times \mathbb{R}_{+}^{d}\right) \subset \operatorname{cone}\left(\operatorname{int} \mathbb{E}\left[\Gamma \mid \mathcal{F}_{t}\right]\right)=: E$ where

$$
\Gamma:=\left\{\left(\gamma_{t+1} y, \bar{M}_{t+1}^{\prime} y+r\right),(y, r) \in\left(K_{t+1}^{*} \cap B_{1}\right) \times[0,1]^{d}\right\} .
$$

If the above does not hold, then $K_{t}^{*} \times \mathbb{R}_{+}^{d} \not \subset \bar{E}$ on a set $A \in \mathcal{F}_{t}$, with $\mathbb{P}[A]>0$, and the same arguments as in step 1 imply that we can find $(p, q) \in L^{0}\left(K_{t}^{*} \times \mathbb{R}_{+}^{d}, \mathcal{F}_{t}\right)$ and $(\zeta, \beta) \in L^{0}\left(\mathbb{R}^{d} \times \mathbb{R}^{d}, \mathcal{F}_{t}\right)$ such that

$p^{\prime} \zeta+q^{\prime} \beta<0$ on $A$ and $0 \leq Y^{\prime}\left(\gamma_{t+1} \zeta+\bar{M}_{t+1} \beta\right)+\rho^{\prime} \beta$ for all $(Y, \rho) \in L^{\infty}\left(K_{t+1}^{*} \times \mathbb{R}_{+}^{d}, \mathcal{F}_{t+1}\right)$.

The right-hand side implies that $\beta \in \mathbb{R}_{+}^{d}$ and $\gamma_{t+1} \zeta+\bar{M}_{t+1} \beta=\gamma_{t+1}\left(\zeta+M_{t+1} \beta\right) \in K_{t+1}$, and therefore $\zeta+M_{t+1} \beta \in K_{t+1}$. In view of $\mathbf{N A 2}{ }^{L}$, this implies that $\zeta \in K_{t}$. On the other hand, the fact that $(q, \beta) \in \mathbb{R}_{+}^{d} \times \mathbb{R}_{+}^{d}$ combined with the left-hand side inequality above implies that $p^{\prime} \zeta<0$. This leads to a contradiction since $p \in K_{t}^{*}$ and $\zeta \in K_{t}$.

4. We can now conclude the proof. Fix $X \in L^{1}\left(\operatorname{int} K_{t}^{*}, \mathcal{F}_{t}\right)$, let $\tilde{Y}$ be as in step 2 and fix $\varepsilon \in L^{1}\left((0,1], \mathcal{F}_{t}\right)$ such that $\tilde{X}:=X-\varepsilon \mathbb{E}\left[\gamma_{t+1} \tilde{Y} \mid \mathcal{F}_{t}\right] \in L^{1}\left(\operatorname{int} K_{t}^{*}, \mathcal{F}_{t}\right)$. It then follows from step 3 and Lemma 4.1 that, for any $\eta \in L^{0}\left(\operatorname{int} \mathbb{R}_{+}^{d}, \mathcal{F}_{t}\right)$, we can find $Y \in L^{\infty}\left(K_{t+1}^{*}, \mathcal{F}_{t+1}\right)$ and $\alpha \in L^{0}\left(\mathbb{R}_{+}, \mathcal{F}_{t}\right)$ such that $\tilde{X}=\mathbb{E}\left[\gamma_{t+1} \alpha Y \mid \mathcal{F}_{t}\right]$ and $\eta-\mathbb{E}\left[\gamma_{t+1} \alpha M_{t+1}^{\prime} Y \mid \mathcal{F}_{t}\right] \in \mathbb{R}_{+}^{d}$. In view of step 2., we can choose $\eta$ such that $\eta+\varepsilon \mathbb{E}\left[\bar{M}_{t+1}^{\prime} \tilde{Y} \mid \mathcal{F}_{t}\right] \in \operatorname{int} \mathbb{R}_{-}^{d}$. This implies that $X=\mathbb{E}\left[Z \mid \mathcal{F}_{t}\right]$ and $\mathbb{E}\left[M_{t+1}^{\prime} Z \mid \mathcal{F}_{t}\right] \in$ $\operatorname{int} \mathbb{R}_{-}^{d}$ where $Z:=\gamma_{t+1}(\alpha Y+\varepsilon \tilde{Y}) \in \operatorname{int} K_{t+1}^{*}$. Since $X \in L^{1}$ and $K^{*} \subset \mathbb{R}_{+}^{d}$, we must have $Z \in L^{1}$. Moreover, $\tilde{Y}, Y$ and $\gamma_{t+1} M_{t+1}=\bar{M}_{t+1}$ are essentially bounded, while $\alpha$ and $\varepsilon$ are $\mathcal{F}_{t}$-measurable, so that $\mathbb{E}\left[\left|M_{t+1}^{\prime} Z\right| \mid \mathcal{F}_{t}\right]<\infty \mathbb{P}-$ a.s. This shows the required result.

It remains to prove the opposite implication of Theorem 2.1.

Proposition 4.2 $\mathrm{PCE}^{L} \Rightarrow \mathrm{NA}^{L}$.

Proof We fix $t<T$.

1. We first assume that we can find $(\zeta, \beta) \in L^{0}\left(\mathbb{R}^{d} \times \mathbb{R}_{+}^{d}, \mathcal{F}_{t}\right)$ satisfying

$$
\zeta-\beta+L_{t+1} \beta \in K_{t+1},
$$


and such that $\zeta \notin K_{t}$ on a set $A \in \mathcal{F}_{t}$ of positive measure. Without loss of generality, we can assume that $(\zeta, \beta) \in L^{\infty}\left(\mathbb{R}^{d} \times \mathbb{R}_{+}^{d}, \mathcal{F}_{t}\right)$, since the same statements hold for $(1+|\zeta|+|\beta|)^{-1}(\zeta, \beta)$. This implies that we can find $Z_{t} \in L^{1}\left(\operatorname{int} K_{t}^{*}, \mathcal{F}_{t}\right)$ such that

$$
Z_{t}^{\prime} \zeta<0 \text { on } A
$$

In view of $\mathbf{P C E} \mathbf{E}^{L}$, we can then find $Z_{t+1} \in L^{1}\left(\operatorname{int} K_{t+1}^{*}, \mathcal{F}_{t+1}\right)$ such that $\mathbb{E}\left[Z_{t+1} \mid \mathcal{F}_{t}\right]=Z_{t}$, $\mathbb{E}\left[\left|Z_{t+1}^{\prime}\left(L_{t+1}-I_{d}\right)\right| \mid \mathcal{F}_{t}\right]<\infty$ and $\mathbb{E}\left[Z_{t+1}^{\prime}\left(L_{t+1}-I_{d}\right) \mid \mathcal{F}_{t}\right] \in \operatorname{int} \mathbb{R}_{-}^{d}$. By (4.2), we have $Z_{t+1}^{\prime} \zeta+Z_{t+1}^{\prime}\left(L_{t+1}-I_{d}\right) \beta \geq 0$ which, by taking conditional expectations, leads to $Z_{t}^{\prime} \zeta+\mathbb{E}\left[Z_{t+1}^{\prime}\left(L_{t+1}-I_{d}\right) \mid \mathcal{F}_{t}\right] \beta \geq 0$. Since $\mathbb{E}\left[Z_{t+1}^{\prime}\left(L_{t+1}-I_{d}\right) \mid \mathcal{F}_{t}\right] \in \operatorname{int} \mathbb{R}_{-}^{d}$ and $\beta \in \mathbb{R}_{+}^{d}$, this leads to a contradiction to (4.3).

2. We now assume that $\beta \in L^{0}\left(\mathbb{R}_{+}^{d}, \mathcal{F}_{t}\right)$ is such that $\left(L_{t+1}-I_{d}\right) \beta \in K_{t+1}$. For $Z_{t+1}$ defined as above, we obtain $Z_{t+1}^{\prime}\left(L_{t+1}-I_{d}\right) \beta \geq 0$ while $\mathbb{E}\left[Z_{t+1}^{\prime}\left(L_{t+1}-I_{d}\right) \mid \mathcal{F}_{t}\right] \in \operatorname{int} \mathbb{R}_{-}^{d}$. This implies that $\beta=0$.

\subsection{The closedness properties}

In this section, we prove that the set $A_{0}^{L}(T)$ is closed in probability whenever there exists a $(K, L)$-strictly consistent price system, i.e., $\mathcal{M}_{0}^{T}\left(\operatorname{int} K^{*}\right) \cap \mathcal{L}_{0}^{T}\left(\operatorname{int} \mathbb{R}_{-}^{d}\right) \neq \emptyset$, and that the same holds for $A_{0}^{R}(T)$ under the additional assumption (USC). In view of Theorem 2.1, Theorem 2.2 is a direct consequence of Corollary 4.1 below. We start with the proof of the key Lemma 2.1 which will be later applied to the linear case $R(\beta)=0+L \beta$.

Proof of Lemma 2.1. Fix $\check{Z}$ such that (2.9) holds. In this proof, we set $M_{t+1}:=$ $L_{t+1}-I_{d}$ and $\bar{Z}_{t}:=\mathbb{E}\left[\check{Z}_{t+1}^{\prime} M_{t+1} \mid \mathcal{F}_{t}\right]$, for $t<T$, in order to clear notations. We first observe that $\left(\check{Z}_{t}, \bar{Z}_{t}\right) \in \operatorname{int} K_{t}^{*} \times \operatorname{int} \mathbb{R}_{-}^{d}$ implies:

$$
\check{Z}_{t}^{\prime} \xi \leq-\varepsilon|\xi| \text { and } \bar{Z}_{t}^{\prime} \beta \leq-\varepsilon|\beta| \text { for all }(\xi, \beta) \in L^{0}\left(\left(-K_{t}\right) \times \mathbb{R}_{+}^{d}, \mathcal{F}_{t}\right), t \leq T,
$$

for some $\varepsilon \in L^{0}((0,1), \mathcal{F})$, compare this also with Lemma 3.1 in [2].

We next deduce from (2.3)-(2.5) that

$V_{T}^{\xi, \beta}=X_{T}$ where $X_{t}:=\sum_{s \leq t} \xi_{s}+\zeta_{s}+\left(c_{s}+M_{s} \beta_{s-1}\right) \mathbf{1}_{s \geq 1}$ for some $\zeta \in L^{0}(-K, \mathbb{F})(4.5)$

Since $X_{T}+\kappa=V_{T}^{\xi, \beta}+\kappa \in K_{T}$, we have $\check{Z}_{T}^{\prime} X_{T} \geq-\check{Z}_{T}^{\prime} \kappa$ so $\mathbb{E}\left[\check{Z}_{T}^{\prime} X_{T} \mid \mathcal{F}_{T-1}\right]$ is well defined, since $\check{Z}_{T} \in L^{1}$. It then follows from the martingale property of $\check{Z}$, (4.4), (2.9) and (4.5) that

$-\check{Z}_{T-1}^{\prime} \kappa \leq \mathbb{E}\left[\check{Z}_{T}^{\prime} X_{T} \mid \mathcal{F}_{T-1}\right] \leq \check{Z}_{T-1}^{\prime} X_{T-1}+\mathbb{E}\left[\check{Z}_{T}^{\prime} C_{T-1}^{T}-\varepsilon\left(\left|\xi_{T}\right|+\left|\zeta_{T}\right|+\left|\beta_{T-1}\right|\right) \mid \mathcal{F}_{T-1}\right]$, 
where $C_{T-1}^{T}$ is defined in (2.10). Iterating this procedure leads to

$$
-\check{Z}_{0}^{\prime} \kappa \leq \mathbb{E}\left[\check{Z}_{T}^{\prime} X_{T}\right] \leq \mathbb{E}\left[\check{Z}_{T} C_{0}^{T}-\varepsilon \sum_{0 \leq t \leq T}\left(\left|\xi_{t}\right|+\left|\zeta_{t}\right|+\left|\beta_{t-1}\right| \mathbf{1}_{t \geq 1}\right)\right]
$$

which implies the required result for $\mathbb{Q} \sim \mathbb{P}$ defined by $d \mathbb{Q} / d \mathbb{P}:=\varepsilon \alpha$ with $\alpha:=1 / \mathbb{E}[\varepsilon]$.

We can now prove the closedness properties.

Corollary 4.1 Assume that there exists $Z \in \mathcal{M}_{0}^{T}\left(\operatorname{int} K^{*}\right) \cap \mathcal{L}_{0}^{T}\left(\operatorname{int} \mathbb{R}_{-}^{d}\right)$. Then, $A_{0}^{L}(T)$ is closed in probability. If moreover (USC) is satisfied, then the same holds for $A_{0}^{R}(T)$.

Proof We use an induction argument which combines the techniques first introduced in [10] and Lemma 2.1 applied to the linear case $R(\beta)=0+L \beta$.

1. We first check that $A_{T}^{R}(T)$ is closed in probability, recall (2.4). Indeed, let $\left(g_{n}\right)_{n \geq 1} \subset$ $A_{T}^{R}(T)$ be such that $g_{n} \rightarrow g \in L^{0}\left(\mathbb{R}^{d}, \mathcal{F}\right) \mathbb{P}-$ a.s. as $n \rightarrow \infty$. Let $\left(\xi_{T}^{n}, \beta_{T}^{n}\right)_{n \geq 1} \in$ $L^{0}\left(\left(-K_{T}\right) \times \mathbb{R}_{+}^{d}, \mathcal{F}_{T}\right)$ be such that $\xi_{T}^{n}-\beta_{T}^{n}=g_{n}$ for all $n \geq 1$ and set $E:=\left\{\liminf _{n \rightarrow \infty}\left|\beta_{T}^{n}\right|<\right.$ $\infty\}$. We claim that $E=\Omega$. Indeed, letting $\left(\bar{\xi}_{T}^{n}, \bar{\beta}_{T}^{n}\right):=\left(\xi_{T}^{n}, \beta_{T}^{n}\right) /\left(1+\left|\beta_{T}^{n}\right|\right) \mathbf{1}_{E^{c}}$, we obtain $\bar{\xi}_{T}^{n}=\mathbf{1}_{E^{c}} g_{n} /\left(1+\left|\beta_{T}^{n}\right|\right)+\bar{\beta}_{T}^{n}$. In view of Lemma 4.2 below, we can assume, after possibly passing to an $\mathcal{F}_{T^{-}}$-measurable subsequence, that $\mathbf{1}_{E^{c}} g_{n} /\left(1+\left|\beta_{T}^{n}\right|\right)+\bar{\beta}_{T}^{n} \rightarrow$ $\bar{\beta}_{T} \in L^{0}\left(\mathbb{R}_{+}^{d}, \mathcal{F}_{T}\right) \mathbb{P}$ - a.s. as $n \rightarrow \infty$, with $\left|\bar{\beta}_{T}\right|=1$ on $E^{c}$. On the other hand $\bar{\xi}_{T}^{n} \mathbf{1}_{E^{c}} \in-K_{T} \mathbf{1}_{E^{c}} \mathbb{P}-$ a.s. Since $-K_{T} \cap \mathbb{R}_{+}^{d}=\{0\}$, this leads to a contradiction. It follows that $\liminf _{n \rightarrow \infty}\left|\beta_{T}^{n}\right|<\infty \mathbb{P}$ - a.s. The closedness property of $A_{T}^{R}(T)$ then follows from Lemma 4.2 again. The fact that $A_{T}^{L}(T)$ is closed in probability follows from the same arguments.

2. We now fix $t<T$, assume that $A_{t+1}^{R}(T)$ and $A_{t+1}^{L}(T)$ are closed in probability and deduce that the same holds for $A_{t}^{R}(T)$. The corresponding result for $A_{t}^{L}(T)$ is obviously obtained by considering the special case where $R(\beta)=0+L \beta$.

Let $\left(g_{n}\right)_{n \geq 1} \subset A_{t}^{R}(T)$ and $\left(\xi^{n}, \beta^{n}\right)_{n \geq 1} \subset \mathcal{A}_{0}$ be such that

$$
V_{T}^{\xi^{n}, \beta^{n}}=g_{n} \text { for all } n \geq 1 \text {. }
$$

We assume that

$$
g_{n} \rightarrow g \in L^{0}\left(\mathbb{R}^{d}, \mathcal{F}\right) \mathbb{P}-\text { a.s. as } n \rightarrow \infty .
$$

In view of $(2.5)$, we can find $\left(V^{n}\right)_{n \geq 1} \subset A_{t+1}^{L}(T)$ such that

$$
\xi_{t}^{n}+\left(L_{t+1}-I_{d}\right) \beta_{t}^{n}+C_{t}^{T}+V^{n}=g_{n},
$$


where $C_{t}^{T}$ has been defined in (2.10). Set $\alpha_{n}:=1+\left|\xi_{t}^{n}\right|+\left|\beta_{t}^{n}\right|$. We claim that $E:=\left\{\liminf _{n \rightarrow \infty} \alpha_{n}<\infty\right\}$ has probability one. Indeed, the previous equality implies that

$$
\bar{\xi}_{t}^{n}+\left(L_{t+1}-I_{d}\right) \bar{\beta}_{t}^{n}+\bar{V}^{n}=\mathbf{1}_{E^{c}}\left(g_{n}-C_{t}^{T}\right) / \alpha_{n}
$$

where $\left(\bar{\xi}_{t}^{n}, \bar{\beta}_{t}^{n}\right):=\mathbf{1}_{E^{c}}\left(\xi_{t}^{n}, \beta_{t}^{n}\right) / \alpha_{n} \in L^{0}\left(\left(-K_{t}\right) \times \mathbb{R}_{+}^{d}, \mathcal{F}_{t}\right)$ and $\bar{V}^{n}:=\mathbf{1}_{E^{c}} V^{n} / \alpha_{n} \in$ $A_{t+1}^{L}(T)$. Moreover, Lemma 4.2 below implies that, after possibly passing to an $\mathcal{F}_{t^{-}}$ measurable subsequence, $\left(\bar{\xi}_{t}^{n}, \bar{\beta}_{t}^{n}\right) \rightarrow\left(\bar{\xi}_{t}, \bar{\beta}_{t}\right) \mathbb{P}-$ a.s. as $n \rightarrow \infty$ for some $\left(\bar{\xi}_{t}, \bar{\beta}_{t}\right) \in$ $L^{0}\left(\left(-K_{t}\right) \times \mathbb{R}_{+}^{d}, \mathcal{F}_{t}\right)$ such that $\left(\bar{\xi}_{t}, \bar{\beta}_{t}\right) \neq 0$ on $E^{c}$. Since $A_{t+1}^{L}(T)$ is closed in probability, it follows that

$\bar{V}^{n}=\mathbf{1}_{E^{c}}\left(g_{n}-C_{t}^{T}\right) / \alpha_{n}-\bar{\xi}_{t}^{n}-\left(L_{t+1}-I_{d}\right) \bar{\beta}_{t}^{n} \rightarrow-\bar{\xi}_{t}-\left(L_{t+1}-I_{d}\right) \bar{\beta}_{t} \in A_{t+1}^{L}(T)$ as $n \rightarrow \infty$.

We can then find $(\xi, \beta) \in \mathcal{A}_{0}$ such that

$$
\bar{\xi}_{t}+\left(L_{t+1}-I_{d}\right) \bar{\beta}_{t}+\sum_{t+1 \leq s \leq T} \xi_{s}+\left(L_{s+1} \mathbf{1}_{s+1 \leq T}-I_{d}\right) \beta_{s}=0 .
$$

We can now appeal to Lemma 2.1 applied to the case $R(\beta)=0+L \beta$ to deduce that $\mathbb{E}^{\mathbb{Q}}\left[\left|\bar{\xi}_{t}\right|+\left|\bar{\beta}_{t}\right|\right] \leq 0$, for some $\mathbb{Q} \sim \mathbb{P}$. Since $\left(\bar{\xi}_{t}, \bar{\beta}_{t}\right) \neq 0$ on $E^{c}$, this implies that $\mathbb{P}\left[E^{c}\right]=0$, and therefore $\liminf _{n \rightarrow \infty} \alpha_{n}<\infty \mathbb{P}-$ a.s. Using Lemma 4.2 below, one can then assume, after possibly passing to an $\mathcal{F}_{t}$-measurable random subsequence, that $\left(\xi_{t}^{n}, \beta_{t}^{n}\right)_{n \geq 1}$ converges $\mathbb{P}-$ a.s. to some $\left(\xi_{t}, \beta_{t}\right) \in L^{0}\left(\left(-K_{t}\right) \times \mathbb{R}_{+}^{d}, \mathcal{F}_{t}\right)$, for all $t \leq T$. Using the semi-continuity assumption (USC) and $d$ iterative applications of Lemma 4.2 , we can then find an $\mathcal{F}_{t+1}$-measurable subsequence $(\sigma(n))_{n \geq 1}$ such that $R_{t+1}\left(\beta_{t}^{\sigma(n)}\right) \rightarrow R_{t+1}\left(\beta_{t}\right)+\zeta_{t+1} \mathbb{P}$ - a.s. as $n \rightarrow \infty$ with $\zeta_{t+1} \in L^{0}\left(-K_{t+1}, \mathcal{F}_{t+1}\right)$. It then follows from (4.7) that

$$
\sum_{t+1 \leq s \leq T}\left(\xi_{s}^{\sigma(n)}+R_{s+1}\left(\beta_{s}^{\sigma(n)}\right) \mathbf{1}_{s+1 \leq T}-\beta_{s}^{\sigma(n)}\right) \rightarrow g-\zeta_{t+1}-\left(\xi_{t}+R_{t+1}\left(\beta_{t}\right)-\beta_{t}\right) \mathbb{P}-\text { a.s. }
$$

We conclude by using the fact that the left-hand side term belongs to $A_{t+1}^{R}(T)$ which is closed in probability by assumption.

We conclude this section with the statement we used in the above proof, see [12].

Lemma 4.2 Fix $t \leq T$ and $\left(\eta^{n}\right)_{n \geq 1} \subset L^{0}\left(\mathbb{R}^{d}, \mathcal{F}_{t}\right)$ be such that $\liminf _{n \rightarrow \infty}\left|\eta^{n}\right|<\infty$. Then, there exists a $\mathbb{P}$ - a.s.-increasing sequence $(\sigma(n))_{n \geq 1} \subset L^{0}\left(\mathbb{N}, \mathcal{F}_{t}\right)$ converging $\mathbb{P}-$ a.s. to $\infty$ such that $\left(\eta^{\sigma(n)}\right)_{n \geq 1}$ converges $\mathbb{P}-$ a.s. 


\subsection{Super-hedging theorems}

We now turn to the proof of the super-hedging theorems, i.e., Propositions 3.1, 3.2 and 3.3. The result of Proposition 3.1 is a consequence of Proposition 3.3 and Remark 3.1. The fact that (i) $\Rightarrow$ (ii) $\Rightarrow$ (iii) in Propositions 3.2 and 3.3 is obvious. In the following, we prove that (iii) $\Rightarrow$ (i) in Propositions 3.2 and 3.3 under the corresponding additional assumptions. The fact that (ii) $\Rightarrow$ (i) is obtained by similar, actually shorter, arguments which are fully contained in what follows.

Proof of $($ iii $) \Rightarrow$ (i) in Proposition 3.2: For ease of notations, we write $M$ for $L-I_{d}$. Fix $V \in L^{0}\left(\mathbb{R}^{d}, \mathcal{F}\right)$ such that $V+\kappa \in K_{T}$ for some $\kappa \in \mathbb{R}^{d}$, and assume that $\mathbb{E}\left[Z_{T}^{\prime} V\right] \leq$ $\alpha^{R}(Z)$ for all $Z \in \mathcal{M}_{0}^{T}\left(\operatorname{int} K^{*}\right)$, but that $V \notin A_{0 b}^{R}(T)$. Then, $A_{0 b}^{R}(T)$ being closed in probability by Theorem 2.2, it follows that, for $k$ large enough (after possibly passing to a subsequence), $V^{k}:=V \mathbf{1}_{|V| \leq k}-\kappa \mathbf{1}_{|V|>k}$ does not belong to $A_{0 b}^{R}(T)$ either but satisfies

$$
\mathbb{E}\left[Z_{T}^{\prime} V^{k}\right] \leq \mathbb{E}\left[Z_{T}^{\prime} V\right] \leq \alpha^{R}(Z) \text { for all } Z \in \mathcal{M}_{0}^{T}\left(\operatorname{int} K^{*}\right)
$$

Since $A_{0}^{R}(T)$ is closed in probability, $A_{0}^{R}(T) \cap L^{1}\left(\mathbb{R}^{d}, \mathcal{F}\right)$ is closed in $L^{1}\left(\mathbb{R}^{d}, \mathcal{F}\right)$. The latter being convex under $(\mathbf{R})(\mathrm{a})$, we deduce from the Hahn-Banach theorem that we can find $Y \in L^{\infty}\left(\mathbb{R}^{d}, \mathcal{F}\right)$ and $r \in \mathbb{R}$ such that

$$
\mathbb{E}\left[Y^{\prime} X\right] \leq r<\mathbb{E}\left[Y^{\prime} V^{k}\right] \text { for all } X \in A_{0}^{R}(T) \cap L^{1}\left(\mathbb{R}^{d}, \mathcal{F}\right) .
$$

Set $Z_{t}^{Y}:=\mathbb{E}\left[Y \mid \mathcal{F}_{t}\right]$. Recalling that $R(0)^{-} \in L^{1}$ under $(\mathbf{R})(\mathrm{b})$, we deduce that any element of the form

$$
X=\xi+\sum_{0<t \leq T}\left(R_{t}^{i}(0) \wedge 1\right)_{i \leq d}, \xi \in L^{1}\left(-K_{s}, \mathcal{F}_{s}\right) \text { for some } s \leq T,
$$

belongs to $A_{0}^{R}(T) \cap L^{1}\left(\mathbb{R}^{d}, \mathcal{F}\right)$. This easily leads to $Z_{s}^{Y} \in K_{s}^{*}$ for $s \leq T$. Fix $\tilde{Z} \in$ $\mathcal{M}_{0}^{T}\left(\operatorname{int} K^{*}\right)$, such that $\alpha^{R}(\tilde{Z})<\infty$, which is possible by assumption, and $\varepsilon \in(0,1)$, so that $\check{Z}:=\varepsilon \tilde{Z}+(1-\varepsilon) Z^{Y} \in \mathcal{M}_{0}^{T}\left(\operatorname{int} K^{*}\right)$ and

$$
\mathbb{E}\left[\check{Z}_{T}^{\prime} X\right] \leq(1-\varepsilon) r+\varepsilon \alpha^{R}(\tilde{Z})<\mathbb{E}\left[\check{Z}_{T}^{\prime} V^{k}\right] \quad \forall X \in A_{0}^{R}(T) \cap L^{\infty}\left(\mathbb{R}^{d}, \mathcal{F}\right) .
$$

In order to conclude the proof, it suffices to show that

$$
\alpha^{R}(Z)=\sup \left\{\mathbb{E}\left[Z_{T}^{\prime} X\right], X \in A_{0}^{R}(T) \cap L^{\infty}\left(\mathbb{R}^{d}, \mathcal{F}\right)\right\}, Z \in \mathcal{M}_{0}^{T}\left(K^{*}\right),
$$

which, combined with (4.9), would imply that $\alpha^{R}(\check{Z})<\mathbb{E}\left[\check{Z}_{T}^{\prime} V^{k}\right]$. Thus leading to a contradiction with (4.8), since $\check{Z} \in \mathcal{M}_{0}^{T}\left(\operatorname{int} K^{*}\right)$. 
To see that the above claim holds, first observe that, for $X \in A_{0}^{R}(T)$ such that $X+\rho \in K_{T}$ for some $\rho \in \mathbb{R}^{d}$, one can always construct an essentially bounded sequence, $X^{n}:=X \mathbf{1}_{|X| \leq n}-\rho \mathbf{1}_{|X|>n}$ for $n \geq 1$, which converges $\mathbb{P}-$ a.s. to $X$. Using Fatou's Lemma, one then obtains $\lim \inf _{n \rightarrow \infty} \mathbb{E}\left[Z_{T}^{\prime} X^{n}\right] \geq \mathbb{E}\left[Z_{T}^{\prime} X\right]$ for all $Z \in \mathcal{M}_{0}^{T}\left(K^{*}\right)$. Moreover, $X+\rho \in K_{T}$ implies $X-X^{n} \in K_{T}$ so that $X^{n} \in A_{0}^{R}(T)$ for all $n \geq 1$. This proves (4.10).

Proof of (iii) $\Rightarrow$ (i) in Proposition 3.3: It suffices to repeat the argument of the above proof with $\tilde{Z} \in \mathcal{L}_{0}^{T}\left(\operatorname{int} \mathbb{R}_{-}^{d}\right)$, which is possible by assumption, and to show that one can choose $Z^{Y}$ such that $\mathbb{E}\left[Z_{t}^{Y^{\prime}}\left(L_{t+1}-I_{d}\right) \mid \mathcal{F}_{t}\right] \in \mathbb{R}_{-}^{d}$ for all $t \leq T$. To see this, recall from the above arguments that $Z^{Y}$ is a martingale and that it satisfies

$$
\mathbb{E}\left[Z_{T}^{Y^{\prime}} X\right] \leq r \text { for all } X \in A_{0}^{R}(T) \cap L^{1}\left(\mathbb{R}^{d}, \mathcal{F}\right),
$$

for some $r \in \mathbb{R}$. It then follows from $(\mathbf{R})(\mathrm{b})$ that

$$
\mathbb{E}\left[Z_{T}^{Y^{\prime}} \sum_{t<T}\left(R_{t+1}^{i}\left(\beta_{t}\right) \wedge n-\beta_{t}^{i}\right)_{i \leq d}\right] \leq r \text { for all } \beta \in L^{\infty}\left(\mathbb{R}_{+}^{d}, \mathbb{F}\right) \text { and } n \geq 1 .
$$

Since $Z_{T}^{Y}$ has non-negative components, as an element of $K_{T}^{*} \subset \mathbb{R}_{+}^{d} \mathbb{P}-$ a.s., the monotone convergence theorem implies that

$$
\mathbb{E}\left[Z_{T}^{Y^{\prime}} \sum_{t<T}\left(R_{t+1}\left(\beta_{t}\right)-\beta_{t}\right)\right] \leq r \text { for all } \beta \in L^{\infty}\left(\mathbb{R}_{+}^{d}, \mathbb{F}\right)
$$

In particular, $(\mathbf{R})(\mathrm{b})$ and the above imply that $Z_{T}^{Y^{\prime}} \sum_{1 \leq t \leq T} R_{t}(0) \in L^{1}$ and that for any $s \leq T-1$

$$
\mathbb{E}\left[Z_{s+1}^{Y}{ }^{\prime}\left(R_{s+1}^{0}\left(\beta_{s}\right)-\beta_{s}\right)\right]+\ell \leq r \quad \text { for all } \beta_{s} \in L^{\infty}\left(\mathbb{R}_{+}^{d}, \mathcal{F}_{s}\right)
$$

where

$$
R^{0}:=R-R(0) \text { and } \ell:=\mathbb{E}\left[Z_{T}^{Y^{\prime}} \sum_{1 \leq t \leq T} R_{t}(0)\right]
$$

Using the first assertion in (R), we then deduce that, for $\eta \geq 1$ and $\beta_{s} \in L^{\infty}\left(\mathbb{R}_{+}^{d}, \mathcal{F}_{s}\right)$,

$$
\begin{aligned}
& R_{s+1}\left(\beta_{s}\right)-\eta^{-1} R_{s+1}(\eta \beta)-\left(1-\eta^{-1}\right) R_{s+1}(0) \\
& =R_{s+1}\left(\eta^{-1} \eta \beta_{s}+\left(1-\eta^{-1}\right) 0\right)-\eta^{-1} R_{s+1}(\eta \beta)-\left(1-\eta^{-1}\right) R_{s+1}(0) \in K_{s+1} .
\end{aligned}
$$


This shows that, for all $\beta_{s} \in L^{\infty}\left(\mathbb{R}_{+}^{d}, \mathcal{F}_{s}\right)$, the sequence $\left(Z_{s+1}^{Y}{ }^{\prime} R_{s+1}^{0}\left(n \beta_{s}\right) / n\right)_{n \geq 1}$ is nonincreasing and that, by (4.11),

$$
\mathbb{E}\left[Z_{s+1}^{Y}{ }^{\prime}\left(R_{s+1}^{0}\left(n \beta_{s}\right) / n-\beta_{s}\right)\right] \leq(r-\ell) / n .
$$

Sending $n \rightarrow \infty$, using the monotone convergence theorem and recalling ( $R \mathbf{L}$ ) leads to

$$
\mathbb{E}\left[Z_{s+1}^{Y}{ }^{\prime}\left(L_{s+1} \beta_{s}-\beta_{s}\right)\right] \leq 0
$$

Since $\beta_{s} \in L^{\infty}\left(\mathbb{R}_{+}^{d}, \mathcal{F}_{s}\right)$ is arbitrary this readily implies that $\mathbb{E}\left[Z_{s+1}^{Y}{ }^{\prime}\left(L_{s+1}-I_{d}\right) \mid \mathcal{F}_{s}\right] \in$ $\mathbb{R}_{-}^{d}$.

\subsection{Utility maximization}

Proof of Proposition 3.4. Let $\left(V^{n}\right)_{n \geq 1}$ be a maximizing sequence. Since $U(V)=$ $-\infty$ on $\left\{V \notin K_{T}\right\}$, it must satisfy $V^{n}+x_{0} \in K_{T}$ for all $n \geq 1$. It then follows from the definition of $\alpha^{R}$ and our assumptions that there exists $Z \in \mathcal{M}_{0}^{T}\left(\operatorname{int} K^{*}\right)$ such that $\mathbb{E}\left[Z_{T}^{\prime}\left(V^{n}+x_{0}\right)\right] \leq \alpha^{R}(Z)+Z_{0}^{\prime} x_{0}<\infty$ for all $n \geq 1$.

Since $Z_{T} \in \operatorname{int} K_{T}^{*}$ and $V^{n}+x_{0} \in K_{T}$, for all $n \geq 1$, we can find $\varepsilon \in L^{0}\left((0,1], \mathcal{F}_{T}\right)$ such that

$$
\mathbb{E}\left[\varepsilon\left|V^{n}+x_{0}\right|\right] \leq \alpha^{R}(Z)+Z_{0}^{\prime} x_{0}<\infty \text { for all } n \geq 1 .
$$

This is similar to Lemma 3.1 in [2]. By Komlos Lemma, one can then find a sequence $\left(\tilde{V}^{n}\right)_{n \geq 1}$ such that $\tilde{V}^{n} \in \operatorname{conv}\left(V^{k}, k \geq n\right)$ for all $n \geq 1$, and $\left(\tilde{V}^{n}\right)_{n \geq 1}$ converges $\mathbb{P}$ - a.s. to some $V\left(x_{0}\right) \in L^{0}\left(\mathbb{R}^{d}, \mathcal{F}\right)$. Since $A_{0}^{R}(T)$ is convex under $(\mathbf{R})(\mathrm{a}),\left(\tilde{V}^{n}\right)_{n \geq 1} \subset$ $A_{0}^{R}(T)$. Since $A_{0}^{R}(T)$ is closed in probability, see Theorem 2.2, we have $V\left(x_{0}\right) \in A_{0}^{R}(T)$. Moreover, the random map $U$ being $\mathbb{P}$ - a.s. concave, $\left(\tilde{V}^{n}\right)_{n \geq 1}$ is also a maximizing sequence. Since $U\left(x_{0}+\tilde{V}^{n}\right)^{+} \leq 1$ for each $n \geq 1$, we finally deduce from Fatou's Lemma and the $\mathbb{P}$ - a.s. upper semi-continuity of $U$ that

$$
\sup _{V \in \mathcal{U}\left(x_{0}\right)} \mathbb{E}\left[U\left(x_{0}+V\right)\right]=\limsup _{n \rightarrow \infty} \mathbb{E}\left[U\left(x_{0}+\tilde{V}^{n}\right)\right] \leq \mathbb{E}\left[U\left(x_{0}+V\left(x_{0}\right)\right)\right] .
$$

\section{References}

[1] B. Bouchard and H. Pham. Optimal consumption in discrete time financial models with industrial investment opportunities and non-linear returns. Annals of Applied Probability, 15(4):2393-2421, 2005. 
[2] L. Campi and W. Schachermayer. A Super-Replication Theorem in Kabanov's Model of Transaction Costs. Finance and Stochastics, 10(4):579-596, 2006.

[3] C. Dellacherie and P.-A. Meyer. Probabilities and Potential. North-Holland, Amsterdam, 1978.

[4] E. Denis and Y. Kabanov. Consistent Price Systems and Arbitrage Opportunities of the Second Kind in Models with Transaction Costs. To appear in Finance and Stochastics, 2010.

[5] J. Grépat and Y. Kabanov. Small transaction costs, absence of arbitrage and consistent price systems. To appear in Finance and Stochastics, 2010.

[6] P. Guasoni, M. Rásonyi and W. Schachermayer. The fundamental theorem of asset pricing for continuous processes under small transaction costs. Annals of Finance, 6 (2):157-191, Springer, 2010.

[7] Y. Kabanov and M. Kijima. A Consumption-Investment Problem with Production Possibilities. From stochastic calculus to mathematical finance: the Shiryaev Festschrift, 315, Springer Verlag, 2006.

[8] Y. Kabanov, M. Rásonyi and C. Stricker. No-arbitrage criteria for financial markets with efficient friction. Finance and Stochastics, 6(3):371-382, 2002.

[9] Y. Kabanov, M. Rásonyi and C. Stricker. On the closedness of sums of convex cones in $L^{0}$ and the robust no-arbitrage property. Finance and Stochastics, 7(3):403-411, 2003.

[10] Y. Kabanov and C. Stricker. A teachers' note on no-arbitrage criteria. Séminaire de Probabilités XXXV, Lect. Notes Math. 1755, Springer, 149-152, 2001.

[11] Y. Kabanov and M. Safarian. Markets with Transaction Costs. Mathematical Theory. Springer-Verlag, 2009.

[12] Y. Kabanov and C. Stricker. A teacher's note on no-arbitrage criteria. Lect. Notes Math., 149-152, Springer Verlag, 2001.

[13] T. Pennanen. Arbitrage and deflators in illiquid markets. Finance and Stochastics, 15(1):57-83, 2011. 
[14] T. Pennanen and I. Penner. Hedging of claims with physical delivery under convex transaction costs. SIAM J. Financial Math., 1:158-178, 2010.

[15] M. Rásonyi. New methods in the arbitrage theory of markets with transaction costs. In: Séminaire de Probabilités, XLI. Lect. Notes in Math., 1934, 455-462, Berlin, 2008.

[16] M. Rásonyi. Arbitrage Under Transaction Costs Revisited. Optimality and RiskModern Trends in Mathematical Finance: The Kabanov Festschrift, 211-225, Springer, 2009.

[17] W. Schachermayer. The fundamental theorem of asset pricing under proportional transaction costs in finite discrete time. Mathematical Finance, 14(1):19-48, 2004. 